\title{
Cell Wall Biochemistry Drives Pollen Tube Mechanics and Affects Growth Rate
}

\author{
Hannes Vogler ${ }^{\mathrm{a}, 1}$, Gautam Munglani ${ }^{\mathrm{a}, \mathrm{b}, 1}$, Tohnyui Ndinyanka Fabrice ${ }^{\mathrm{a}, 2}$, \\ Christian Draeger ${ }^{\mathrm{a}}$, Jan T. Burri ${ }^{\mathrm{c}}$, Christof Eichenberger ${ }^{\mathrm{a}}$, J. Paul Knox ${ }^{\mathrm{d}}$, \\ Jean Claude Mollet ${ }^{\mathrm{e}}$, Bradley J. Nelson ${ }^{\mathrm{c}}$, Hans J. Herrmann ${ }^{\mathrm{b}}$, Christoph \\ Ringli ${ }^{\mathrm{a}, *}$, Ueli Grossniklaus ${ }^{\mathrm{a}, 3, *}$ \\ ${ }^{a}$ Department of Plant and Microbial Biology and Zurich-Basel Plant Science Center, \\ University of Zurich, Zollikerstrasse 107, 8008 Zurich, Switzerland \\ ${ }^{b}$ Institute for Building Materials, ETH Zurich, Wolfgang-Pauli Strasse 27, 8093 Zurich, \\ Switzerland \\ ${ }^{c}$ Department of Mechanical and Process Engineering, ETH Zurich, Tannenstrasse 3, \\ 8092 Zurich, Switzerland \\ ${ }^{d}$ Centre for Plant Sciences, Faculty of Biological Sciences, University of Leeds, Leeds, \\ LS2 9JT, United Kingdom \\ ${ }^{e}$ Normandie Univ, UNIROUEN, Glyco-MEV, SFR NORVEGE, Carnot I2C, 76000 \\ Rouen, France
}

\section{SUMMARY}

Pollen tubes live a life on a razor's edge. They must maintain cell wall integrity whilst growing towards the ovule at extraordinary speed but explosively burst at just the right moment to release the sperm cells-with fatal consequences for reproduction if things go wrong. The precisely controlled growth of the pollen tube depends on the fine-tuned balance between the expansive force of turgor pressure and the restraining effect of the cell

\footnotetext{
*Correspondance: Ueli Grossniklaus, Christoph Ringli

Email addresses: chringli@botinst.uzh.ch (Christoph Ringli), grossnik@botinst.uzh.ch (Ueli Grossniklaus)

${ }^{1}$ These authors contributed equally

${ }^{2}$ Present address: Biozentrum, University of Basel, Klingelbergstrasse 50/70, 4056 Basel, Switzerland

${ }^{3}$ Lead Contact
} 
wall. Currently, it is not well understood how the composition of the cell wall affects its mechanical properties. Using Arabidopsis mutants, we have investigated these interactions by combining experimental and simulation techniques to determine instantaneous and time-dependent mechanical parameters. This allowed, for the first time, the quantification of the effects of cell wall biochemistry on turgor pressure and cell wall elasticity and to predict their effects on growth rate. Our systems biology approach is widely applicable to study the implications of mechanical stress on growth.

Keywords: Arabidopsis thaliana, cellular growth, cell wall mechanics, cellular force microscopy, CFM, finite element modelling, FEM, pollen tube

\section{INTRODUCTION}

In all organisms, mechanical forces and physical constraints influence cellular growth and the development of tissues and, hence, present an essential parameter influencing tissue and organ formation (Mammoto et al., 2013). The mechanical forces that act on a cell can influence its differentiation, physiology, migration, and communication. Different cell types and tissues react in a distinct manner to mechanical influences, which can result in specific functional changes, such that mechanical forces play an important role in development, health, and disease (Argentati et al., 2019; Hayward et al., 2021).

Plant morphogenesis is largely regulated by mechanical equilibrium, as controlled cellular growth involves a finely tuned interplay between turgor pressure as the driving force and the constraining action of the cell wall (Hamant, 2013; Routier-Kierzkowska and Smith, 2013), with the rate of ex- 
pansion being controlled by local changes in cell wall viscosity (Campas and Mahadevan, 2009; Hepler et al., 2013; Cosgrove, 2016). The importance for a tight control of this interaction is particularly evident in tip-growing cells such as pollen tubes (PTs), which are arguably the fastest-expanding cells on the planet. Maize PTs, for example, reach the most distant ovules, up to $30 \mathrm{~cm}$ away from the stigma, in less than $24 \mathrm{~h}$, thus growing at a speed of more than $3 \mathrm{\mu ms}^{-1}$ (Bedinger, 1992). While a tight control of cell wall integrity is essential during the growth phase, the final step - the release of the sperm cells to effect double fertilization-requires rupture of the PT at just the right time and place for successful reproduction. Therefore, PTs lead an existence on a razor's edge with fatal consequences for fertilisation, and thus the production of seeds, the main source for food, feed, fuel, and fibres, if things go wrong.

To gain deeper insights into the delicate relationship between turgor pressure and the mechanical properties of the cell wall, we need to learn more about the effect of biochemical changes in the cell wall on these parameters. While turgor pressure is a well-studied quantity, the correlation between the cell wall's composition and its mechanical properties remains poorly understood. Cell walls are complex supramolecular structures of intertwined polysaccharides and structural proteins. The classical model of the cell wall assumes a network of cellulose microfibrils connected by hemicelluloses that is embedded in a gel-like pectin matrix (Cosgrove, 2016) and re-inforced by structural proteins, such as the highly glycosylated extensins (Showalter and Basu, 2016). Together with callose, all these components were shown to also be present in pollen tube cell walls (Dardelle et al., 2010). The spatially 
and temporally controlled expansion of the cell wall is facilitated by local modifications of its composition, leading to rheological adjustments that allow the cell to adapt its size and shape to specific requirements (Winship et al., 2010). Thus, information on bulk material behavior under varied conditions is required to understand the cell wall's role in regulating cell expansion. In particular, capturing the material response to both short and long timescale loading modalities, yielding time-independent (instantaneous) and time-dependent (relaxation) properties, respectively, is essential to quantifying the competing forces responsible for maintaining mechanical equilibrium and defining cell shape. The instantaneous pliability of the cell is best quantified by its apparent stiffness (a measure of perceived stiffness): a combination of geometric properties, e.g., cell wall thickness, and intrinsic material properties, such as the elastic modulus (a measure of elasticity). In contrast, time-dependent properties, which condition the delayed response of the cell to forces applied and maintained over a longer timescale, are best represented by the cell wall's viscosity.

A range of methods have been developed to measure the material properties of the cell wall, with indentation techniques providing high spatial resolution and, thus, highly localized information in a non-invasive fashion (Routier-Kierzkowska and Smith, 2013; Vogler et al., 2015; Geitmann and Parre, 2004; Hayot et al., 2012). Atomic Force Microscopy (AFM) and Scanning Acoustic Microscopy (SAM) have previously been used to extract information on cell wall components at the submicron scale; however, due to their limited scanning area, they are not well suited to capture global properties of a cell (Milani et al., 2011; Peaucelle et al., 2011). Cellular Force 
Microscopy (CFM), however, is a high-resolution microindentation system specifically developed to measure global mechanical properties of living and growing cells (Felekis et al., 2011). Independent of the type of loading placed on a cell, this technique yields the force measured by the probe on the cell wall as a function of the measured indentation depth. The apparent stiffness of the cell can then be directly extracted from the force-indentation curve obtained from short timescale indentation experiments by evaluating the slope of its linear fit (Figure 2). In combination with geometric parameters, such as the cell diameter before and after plasmolysis and the thickness of the cell wall determined by Transmission Electron Microscopy (TEM), mechanical parameters, e.g. elastic modulus and turgor pressure, can be extracted using Finite Element Method (FEM)-based inverse modelling (Vogler et al., 2013). Alternatively, relaxation experiments at longer timescales yield force versus time curves that can be fit to obtain time-dependent material information, such as viscosity (Figure 2). The main challenge, however, lies in closing the loop by relating such mechanical properties to the biochemical composition of the cell wall in order to explain and predict cellular morphogenesis.

Here, we achieve this goal by combining short and long timescale indentation experiments on PTs of several cell wall mutants in the plant model Arabidopsis thaliana. PTs are a well characterized system to investigate cytomechanical properties. Employing the extensive mutant collections in Arabidopsis allowed us to correlate variation in the biochemical composition of the cell wall with changes in its mechanical properties and PT morphogenesis. We specifically investigated the effects of components affecting xyloglucan $(\mathrm{XyG})$ and pectin polysaccharides, as well as extensins, a family 
of cell wall proteins. XyGs are important for the formation of tight associations with cellulose microfibrils, so called 'biomechanical hot spots' (Park and Cosgrove, 2012a, 2015). The regulation of this network alters the dynamics of cell wall elastoplasticity, modulates its extensibility, and allows for controlled cell growth (Thompson, 2005; Park and Cosgrove, 2012b; Eckardt, 2008). The PT cell wall is somewhat special in its composition, as it consists of a very high proportion of pectins compared to the cell wall of other cell types; in the PT tip, it is the predominant cell wall polysaccharide (Bosch et al., 2005; Chebli and Geitmann, 2007). Newly synthesized pectins are methylesterified when they are secreted at the tip. De-esterification mediated by pectin methylesterases (PMEs) and subsequent crosslinking of acidic pectins by $\mathrm{Ca}^{2+}$ reinforce the subapical PT cell wall and play an important role in viscoelasticity (Ngouémazong et al., 2012; Peaucelle et al., 2015; Altartouri et al., 2019). Pectins are also involved in tethering cellulose microfibrils (Dyson et al., 2012). Taken together, they are proposed to be a key determinant in cell wall loosening during cell expansion. Extensins form long rods that make crosslinks with other cell wall components (Cannon et al., 2008). While originally thought to have a largely structural function in the cell wall, it was recently shown that extensins play a fundamental role in cell wall integrity signalling (Gille et al., 2009; Mecchia et al., 2017; Ndinyanka Fabrice et al., 2018; Sede et al., 2018).

We employed CFM in combination with FEM-based modelling and a Monte Carlo uncertainty quantification approach to assess turgor pressure and cell wall elasticity in growing mutant PTs. Surprisingly, alterations in cell wall biochemistry not only affected the mechanical properties of the cell 
wall but also induced changes in turgor pressure. Depending on the nature of the biochemical changes, these parameters were affected differently. Interested in how these changes translate to changes in PT growth, we took this investigation a step further and developed a method to predict the growth rate of PTs based on their cellular mechanical parameters. Since PT growth is driven by time-varying properties, predicting its growth rate requires the measurement of the viscoelastic properties of the cell wall. Using our combined approach, we were able to show that changes in cell wall biochemistry directly affect growth-controlling parameters such as turgor pressure and cell wall elasticity. This has an immediate impact on the growth rate of mutant PTs, which we were able to accurately predict and experimentally confirm.

\section{RESULTS}

\subsection{Pollen tubes of cell wall mutants have severe growth defects}

Under the assumption that mutations in major cell wall components will upset the balance between turgor pressure and cell wall stiffness leading to destabilized PT growth, we analyzed the PT growth phenotypes of different Arabidopsis mutants. The first line we looked at is deficient in XyG and carries mutant alleles of both XYLOGLUCAN 6-XYLOSYLTRANSFERASE1 (XXT1) and XXT2 (Cavalier et al., 2008). We also analysed the xyloglucanase113 (xeg113) mutant, which interferes with the arabinosylation of extensins; a functionally relevant feature of these proteins (Gille et al., 2009). Extensins potentially play a stabilizing role in the absence of XyG (Kim et al., 2020). Given this possible functional relationship, we also analyzed PTs of the $x x t 1$ xxt2 xeg113 triple mutant. Finally, since neutral domains 
of pectins have been suggested to tether cellulose microfibrils (Zykwinska et al., 2005) and, thus, have a wall stabilizing function, particularly in the absence of XyG (Park and Cosgrove, 2015), we also included the pectin methylesterase 48 (pme48) mutant in our studies. The pme 48 mutant affects the de-methylesterification of pectic homogalacturonans (HGs) and, hence, the rigidification of the cell wall through $\mathrm{Ca}^{2+}$-crosslinking of HGs (Bidhendi and Geitmann, 2016). A previous study reported high frequencies of morphological abnormalities in pme 48 PTs, including bifurcated PTs with additional tip-growing foci and bursting PT tips (Leroux et al., 2015), reminiscent of the phenotypes we found in xxt1 xxt2 and xeg113 PTs (Figure 1).

An examination of the phenotypes caused by changes in cell wall composition revealed a higher germination rate in pollen lacking XyG (xxt1 xxt2 and xxt1 xxt2 xeg113) compared to the wild type (for details, see Table S1). We also observed a significantly higher proportion of burst PTs in these mutant lines (Figure 1A). Among the PTs that were still growing, we found that mutant PTs, especially xxt1 xxt2 xeg113, exhibited irregular growth patterns, such as swelling or branching, more frequently than the wild type, which typically formed long, regularly shaped PTs when germinated in liquid pollen tube growth medium (PTGM) (Figures $1 \mathrm{~A}$ to $1 \mathrm{H})$. The XyG-deficient $x x t 1$ xxt2 PTs had a stronger tendency for tip swelling than the extensin mutant xeg113. The highest frequency of defects, however, was found in the triple mutant xxt1 xxt2 xeg113, in which both XyG levels and extensin function are impaired, and $25 \%$ of the PTs had swollen tips (Figure 1A; Table S1). Tip swelling was not always fatal for the PTs because, often, a new tip formed on the club-like, swollen bulge that replaced the original tip, resulting in a 
temporary resumption of normal growth (Figures 1C to 1E). Especially in pme 48 PTs, tip swelling occurred periodically, leading to local thickenings in otherwise normal-looking PTs (Figure 1D). Branching was relatively rare but, again, occured at higher frequency in xxt1 xxt2 xeg113 than in the other mutants (Figures $1 \mathrm{~A}$ and $1 \mathrm{H}$ ). The observed irregular growth phenotypes indicate a disturbed balance between turgor pressure and cell wall stiffness, resulting in a strong tendency to burst (Figures $1 \mathrm{~A}$ and $1 \mathrm{~F}$ ). This is further supported by the fact that the highest bursting rate of $43 \%$ was observed in the mutant disrupting multiple cell wall components, xxt1 xxt2 xeg113.

To determine how the different mutations altered the ultrastructure of the PT cell wall, we used TEM to examine cross sections of PTs grown in a semi in vivo system. The cell wall of wild-type PTs consisted of a dark, fibrous outer layer and a lighter inner layer (Ndinyanka Fabrice et al., 2017). A similar architecture was observed in the mutant PTs; however, in pme 48

Figure 1 (on the next page). Phenotypes of wild-type and mutant pollen tubes

(A) Quantitative representation of pollen germination and the phenotypes depicted in (B-H).The pme 48 mutant is not shown because the experiment was performed separately and cannot be directly compared. For details see Table S1.

(B) Typical wild-type Arabidopsis PT.

(C-H) Aberrant phenotypes in mutant PTs, such as swelling (C-E), bursting (F), releasing cytoplasmic content (C and $\mathrm{G}$, arrowheads), or branching $(\mathrm{H})$. Tip swelling was often followed by the formation of a new tip, which grew more or less normally (arrows). (C and $\mathrm{F}$ ) xxt1 xxt2, (D) pme 48, (E and $\mathrm{H})$ xeg113, (G) xxt1 xxt2 xeg113.

(I and J) Cell wall structure of wild-type and mutant PTs. (I) Ultrastructure of transverse sections of wild-type and mutant PTs displaying an outer (dark) and an inner (lighter) layer. Cell wall thickness varies in wild-type and mutant PTs (see also Table 1). The inner wall layer in the xxt1 xxt2 and xxt1 xxt2 xeg113 mutants is thickened. In addition, the triple mutant contains clusters of electron-dense material sandwiched between the two cell wall layers. (J) Immunogold labelling (arrowheads point to gold particles) showing a higher accumulation of callose in the inner cell wall of $x x t 1$ xxt2 PTs compared to the wild type. Scale bars: $10 \mu \mathrm{m}$ in (B-H), $1 \mu \mathrm{m}$ in (I), $200 \mathrm{~nm}$ in $(\mathrm{J})$. 
bioRxiv preprint doi: https://doi.org/10.1101/2021.11.09.467870; this version posted November 11, 2021. The copyright holder for this preprint (which was not certified by peer review) is the author/funder. All rights reserved. No reuse allowed without permission.
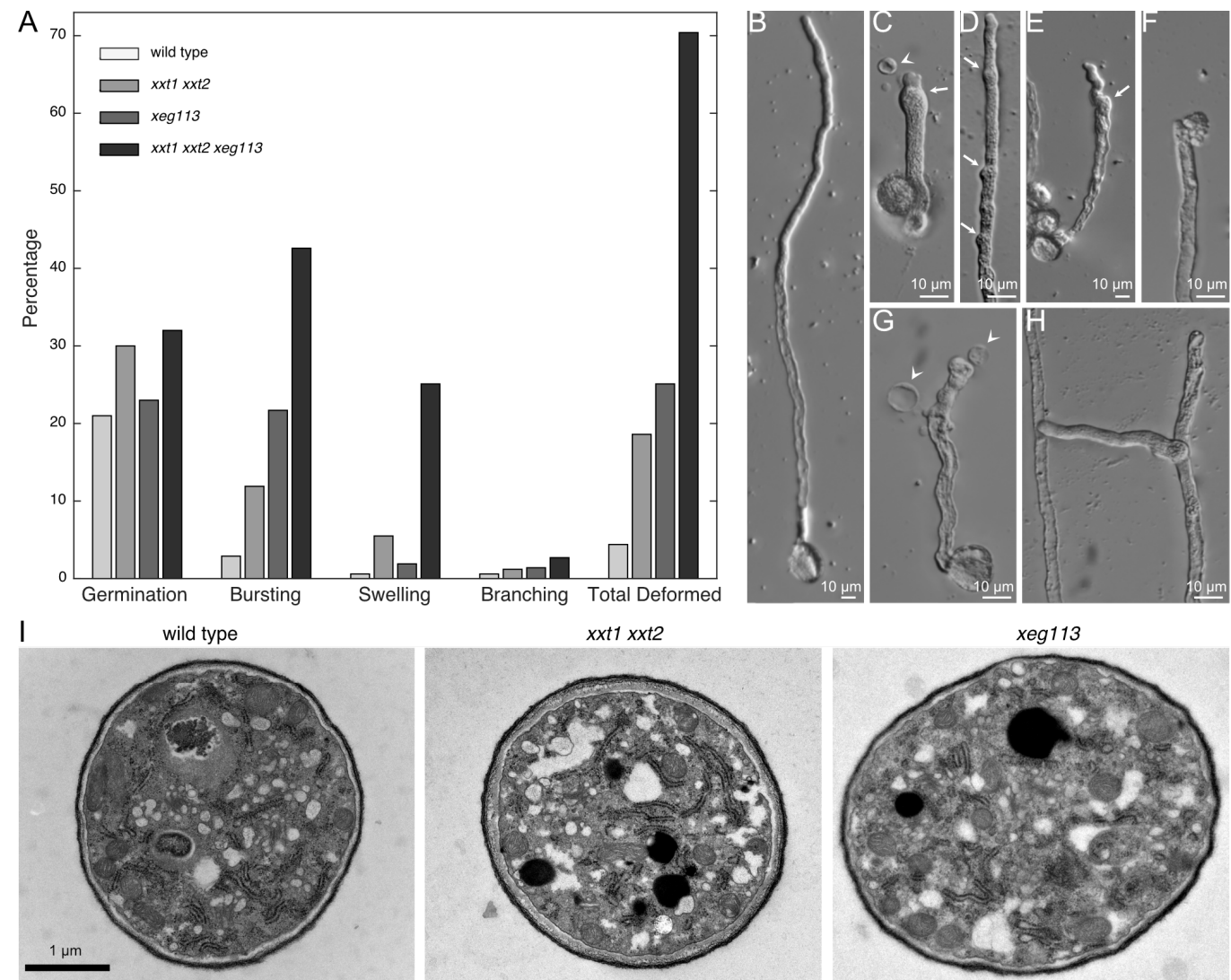
$x x+1 x+2$

xeg113
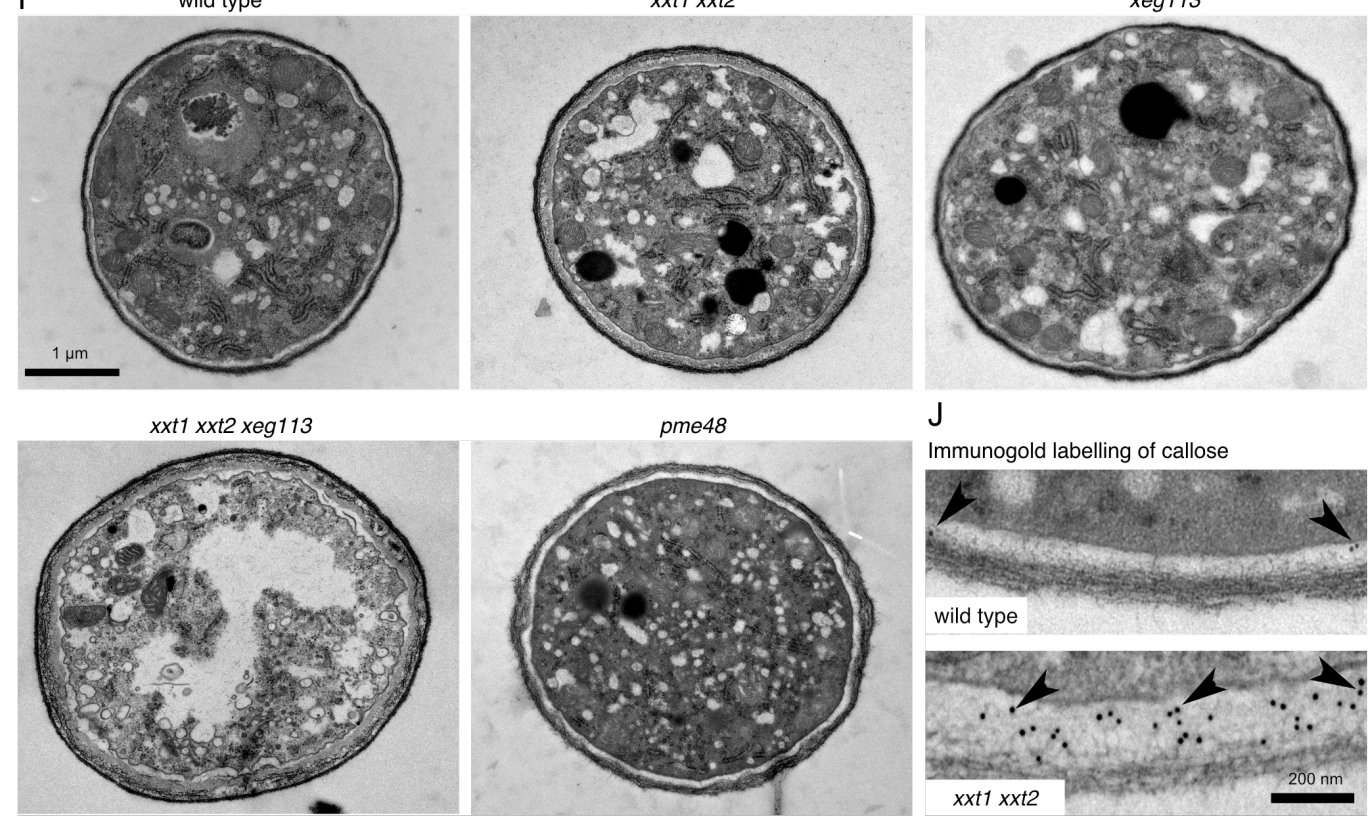

$\mathrm{J}$

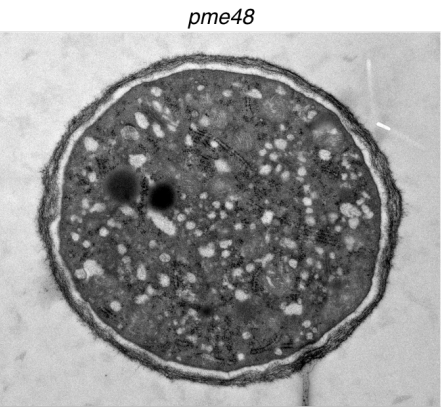

Immunogold labelling of callose
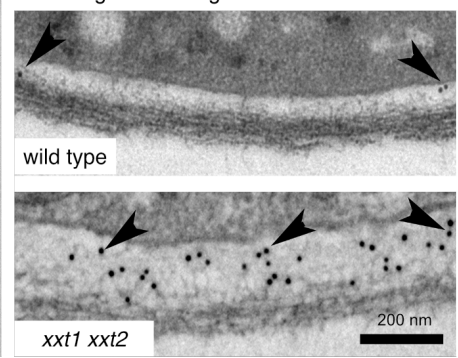
mutants, the outer layer was less dark and the fibrous material seemed a bit less tightly packed, whereas in xxt1 xxt2 and xxt1 xxt2 xeg113 mutants, the inner layer was significantly thicker than in the wild type (Fig. 1I). The inner cell walls of xxt1 xxt2 and xxt1 xxt2 xeg113 PTs are reinforced by increased callose deposition, as revealed by immunogold labelling of TEM sections (Figure 1J), corroborating aniline blue staining results in xxt1 xxt2 mutants (Fig. S1). Furthermore, in xxt1 xxt2 xeg113 PTs, sandwiched between the inner and outer cell wall was a region of varying electron density, with occasional clusters of electron-dense materials, indicative of major defects in both the composition and architecture of the cell wall (Figure 1I). Given the observed differences in callose accumulation, the susceptibility of the various mutant PTs to callose degradation was investigated. Increasing concentrations of the callose-degrading enzyme lyticase (Parre and Geitmann, 2005) resulted in reduced PT growth, with a clearly stronger sensitivity of xxt1 xxt2 and xxt1 xxt2 xeg113 PTs (Fig. S2). This result demonstrates the relevance of callose for Arabidopsis PT growth, where it can compensate for the absence of XyG in the cell wall of mutant PTs.

\subsection{Quantification of mechanical properties in wild-type and mutant pollen tubes}

To elucidate the relationship between the biochemical composition of the cell wall and mechanical properties regulating the observed compensatory mechanisms resulting in growth abnormalities, several input parameters were experimentally measured for subsequent numerical simulations (Figure 2A). These parameters include apparent stiffness, cell wall thickness of the turgid PT, and the diameters of turgid and plasmolysed PTs (Figure 3A and 3B; 
Table 1). CFM experiments revealed that the apparent stiffness showed large variability for all mutant lines except pme48, which also displayed significantly lower values (Figure 3A). Cell wall thickness, a critical parameter that determines how much tensional stress can be withstood at a given stiffness, was revealed to be similar in wild-type and xeg113 PTs. All other mutants, however, had values that were significantly higher than those of the wild type, whilst diverging significantly from each other. Since the cell wall of a turgid PT is constantly under tension due to its internal turgor pressure, PT plasmolysis leads to significant radial shrinkage. The magnitude of this shrinkage acts as a proxy to characterize the elastic behavior of the PT's

$\overline{\text { Figure } 2 \text { (on the next page). Methodological workflow to predict the pollen }}$ tube growth rate

(A, left) CFM, TEM, and light microscopy (LM) experiments to measure the apparent stiffness, cell wall thickness, and the diameter of turgid/plasmolyzed PTs for each variant (wild type and mutants), to which univariate probability distributions are fit. (Right) Stress relaxation experiments using the CFM are performed to extract the normalized force decay in the PT variants over $5 \mathrm{~s}$.

(B) Using Latin hypercube sampling, 1000 samples are drawn from the apparent stiffness, cell wall thickness, and turgid/plasmolyzed PT diameter probability distributions for each PT variant (Iman et al., 1981).

(C) A correlation matrix between the apparent stiffness, cell wall thickness, and turgid/plasmolyzed PT diameters is created using inverse FEM modelling of random conformations of the indentation process. This correlation matrix is then used to correlate the samples drawn from the parameter probability distributions to find correlated sets of parameters th are physically possible in the PT system (Iman and Conover, 1982).

(D) For uncertainty quantification, the correlated sets of parameters are provided as inputs to the FEM-based model where at least 150 correlated parameter sets are simulated per PT variant.

(E) The output turgor pressure and elastic modulus distributions for each PT variant are used as inputs in the growth equation (Eq. 5) to predict the PT growth rate.

(F) The normalized relaxation modulus decay is estimated from the force decay measurements for all PT variants using equation Eq. 1.

(G) Fitting the relaxation modulus decay measurements with equation Eq. 2 yields the cell wall viscosity of the $\mathrm{PT}$ variants, which are coupled with the time-independent parameters as inputs in the growth equation. 


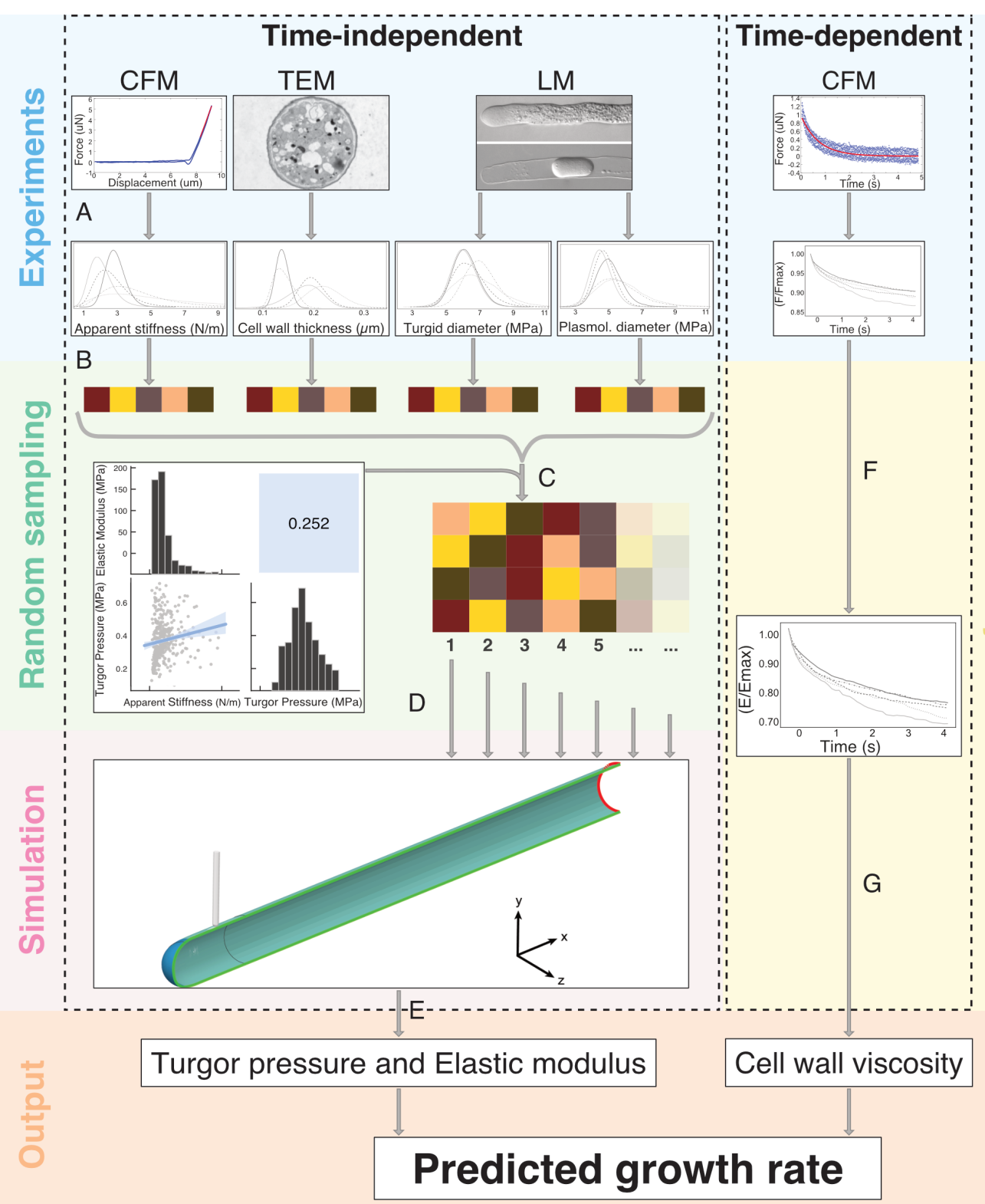


cell wall (Vogler et al., 2013). Wild-type and xxt1 xxt2 PTs displayed similar turgid diameters, while xeg113 and xxt1 xxt2 xeg113 mutant PTs exhibited significantly larger and pme48 PTs significantly lower diameters, respectively (Figure 3B). For plasmolysed diameters, only xxt1 xxt2 xeg113 and pme 48 mutant PTs showed significantly higher and lower values than the wild type, respectively. These measured parameters were sequentially fit to yield the elastic modulus and turgor pressure of the PTs (Figures 3C to 3E), using an inverse continuum FEM-based model of CFM indentation (Krichevsky et al., 2007).

\subsection{Modelling mechanical input parameters}

Due to large intra-sample variance and skew in the experimentally determined input parameters, an elaborate Monte Carlo uncertainty quantification was performed within the FEM framework, starting with a parametric fit on all experimental observations (Fig. 2B). The turgid and plasmolysed PT diameters were best fit using a lognormal distribution due to a strong bias in their lower tails. This skewed distribution is expected, especially among mutant PTs, as smaller diameters indicate greater wall stress, which possibly resulted in the elevated frequency of PTs bursting before measurement. This is supported by previous studies where measurements of biological structures exhibiting growth have also been shown to follow the lognormal distribution (Iman et al., 1981; Koch, 1966; Limpert et al., 2001; Iman and Conover, 1982). These fitted probability distributions were then sampled with Latin hypercube sampling (Iman et al., 1981), and the resulting samples were correlated using the Iman-Conover method (Iman and Conover, 1982) (Figure 2C). Input parameter realizations from these correlated distributions were sub- 


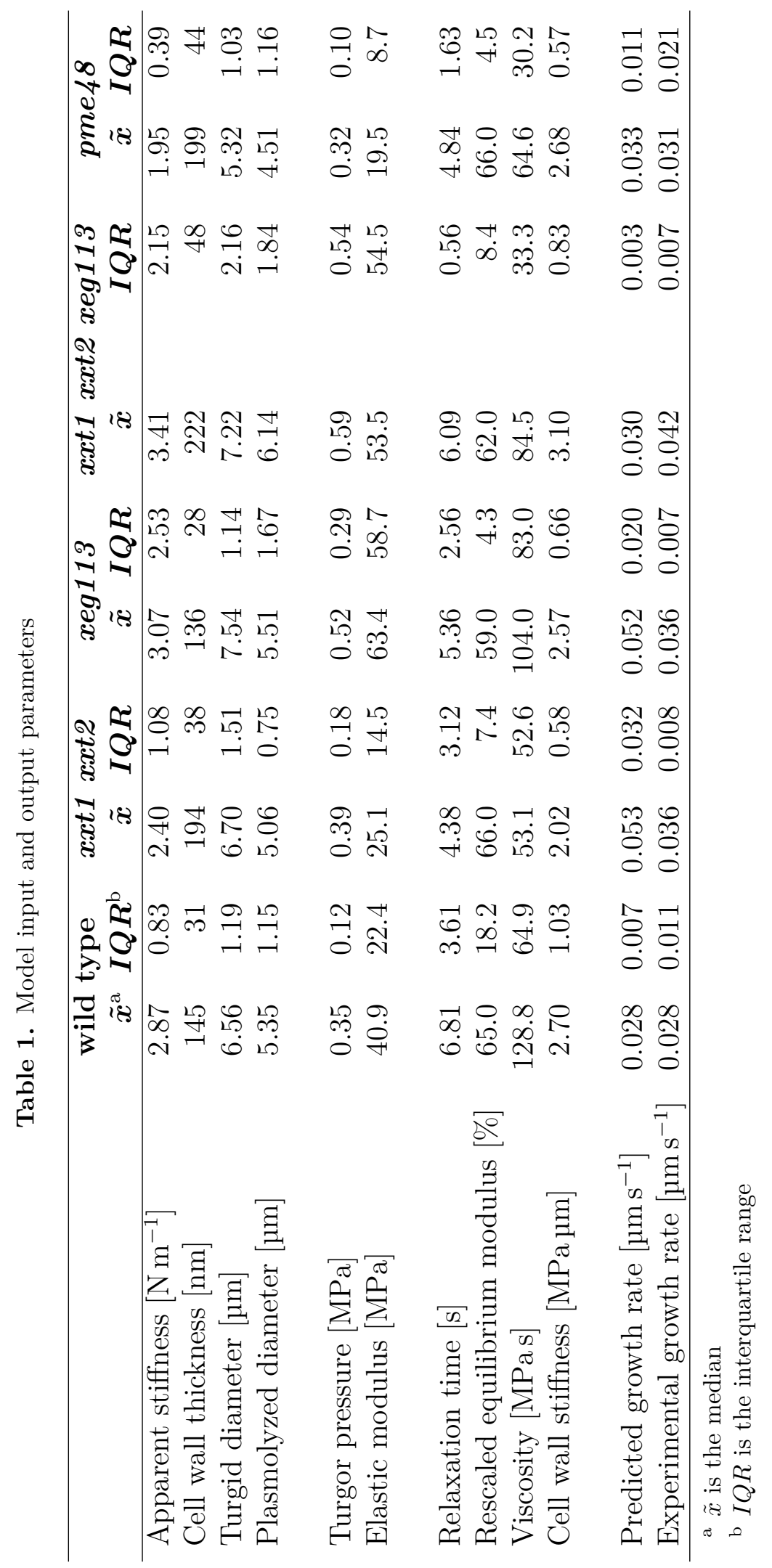


sequently simulated until convergence (minimum 100 realizations) for each mutant using the inverse FEM-based model, until the averaged PT elastic properties - turgor pressure and elastic modulus - could be extracted (Figures $2 \mathrm{D}$ to $2 \mathrm{E})$.

Since a large number of input realizations had to be simulated to obtain accurate statistics for turgor pressure and elastic modulus, the FEM-based model was set up to ensure robustness and computational efficiency, whilst not sacrificing on accuracy. The FEM mesh consisted of a cylindrical shell attached to a hemispherical tip with an imposed pressure boundary condition on the inside wall (total length: $100 \mu \mathrm{m}$ ), constrained by a pinned distal edge. The computational expenditure of the simulations were considerably reduced by cutting the PT in half and enforcing mirror boundary conditions. Since the ratio of PT diameter to cell wall thickness in Arabidopsis is usually less than 25, the shear stress between the inner and outer surfaces cannot be neglected, requiring the use of quadratic brick elements unlike in previous studies on lily PTs (Hearn, 2001; Vogler et al., 2013). The PT cell wall material was characterized using the Saint Venant-Kirchhoff hyperelastic material model, in which the Poisson ratios were set to 0.2 after a sensitivity analysis had shown that varying it in the range between 0.0 and 0.4 had a negligible effect on the apparent stiffness. PT cell walls have also been hypothesized to possess anisotropic properties due to the preferential orientation of cellulose microfibrils (Baskin, 2005). However, a previous CFM study (Vogler et al., 2013) concluded that the PT cell wall possesses only a very slight degree of anisotropy, which is largely masked by the effects of geometry and turgor pressure. Based on this conclusion, the current model neglects the effect of 
anisotropic material properties, as the geometry and range of applied turgor pressure remain unchanged. The contact indentation between the PT and the rigid microelectromechanical systems (MEMS)-based force sensor probe was considered frictionless with finite sliding. It was simulated using a surfaceto-surface discretization with constraints enforced by Lagrange multipliers, along with appropriate damping to stabilize rigid body modes.

The model output showed that the xeg113 and xxt1 xxt2 xeg113 turgor pressure values were significantly elevated, while the turgor pressure in the pme 48 mutant was lower than in wild-type PTs. Furthermore, the elastic moduli of xxt1 xxt2 and pme48 PTs were significantly lower than that of the wild type, while those of xeg113 and xxt1 xxt2 xeg113 PTs were significantly higher. The turgor pressure and elastic modulus interquartile ranges (IQR, $\left.x_{25}-x_{75}\right)$ for $x e g 113$ and even more so for xxt1 xxt2 xeg113 PTs doubled compared to the wild type (Figure 3C), indicating a disruption of the finetuned regulation of cellular morphogenesis. Similarly, the lowered apparent stiffness, turgor pressure, and elastic modulus of pme 48 PTs fit well with the predicted effect of a less rigid cell wall resulting from reduced HG deesterification.

2.4. Principal Component Analysis (PCA) indicates that apparent stiffness, stretch ratio, and cell wall thickness are key parameters of pollen tube growth

As the correlations between input and output parameters were a priori unknown, random realizations of all parameters in the IQR $\left(x_{25}-x_{75}\right)$ were simulated to obtain a correlogram (Figure 4A). Clearly, the apparent stiffness was more correlated to turgor pressure than any other parameter, indicating 
A

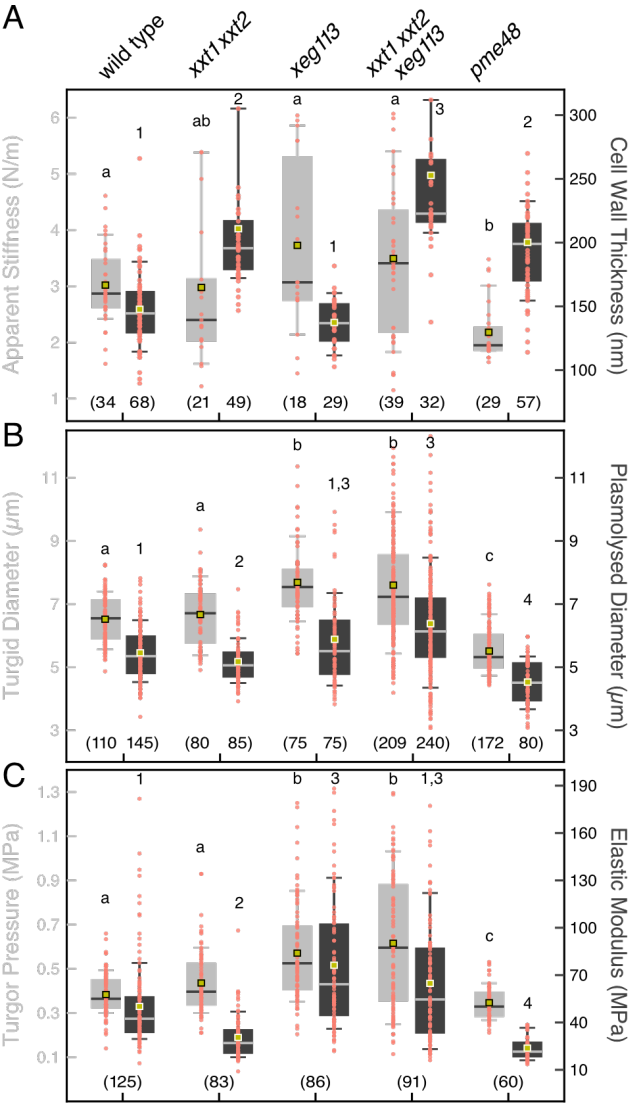

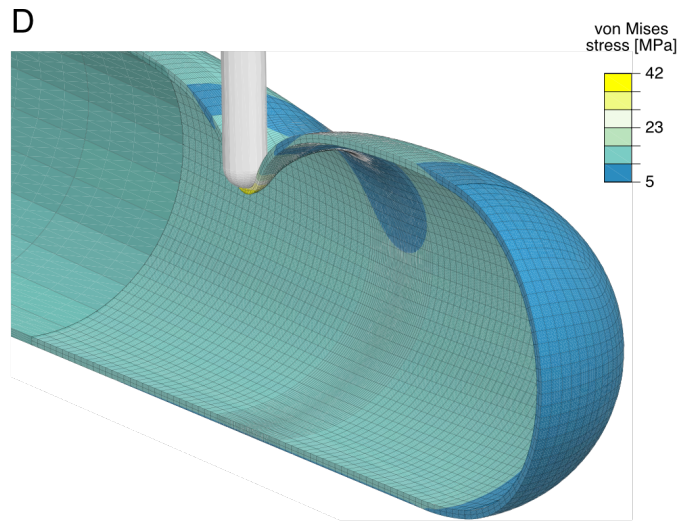

E

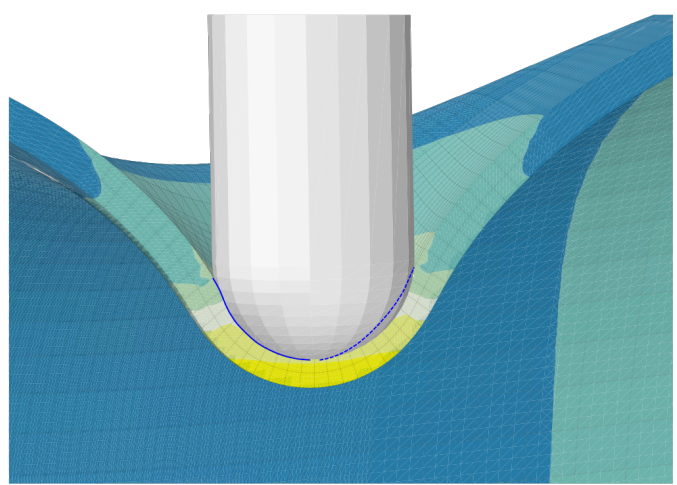

Figure 3. Simulated mechanical properties of wild-type and mutant pollen tubes.

(A) Boxplot of apparent stiffness and cell wall thickness of wild-type and mutant PTs measured by the CFM and TEM, respectively.

(B) Boxplot of diameters in plasmolysed and turgid PTs measured with the LM.

(C) Boxplot of the output turgor pressure and elastic modulus of wild-type and mutant PTs from the FEM/Monte Carlo simulations. The whiskers in A-C represent the data between the $10^{\text {th }}$ and $90^{\text {th }}$ percentiles. The number of analyzed samples is provided at the bottom of each plot, while significance indicators are located at the top. Medians not sharing a common letter (or number) are significantly different from each other.

(D) Finite element mesh of the PT, consisting of a hollow tube constrained to a hemispherical tip, in contact with the rigid indenter of the CFM. A half model is used for computational efficiency. Colors indicate the distribution of von Mises stress caused by the indentation. Mesh density is highest in the indented section to resolve the contact problem and get a high-resolution stress distribution.

(E) Close-up of the indented area. The von Mises stress is highest directly underneath the indenter and reduces drastically with distance from the indenter tip. The contact boundary between the indenter and the PT cell wall is indicated by a blue line (dashed where the indenter masks the contact area). 
that the CFM technique is most sensitive to fluctuations in turgor pressure at a calibrated indentation depth and osmolarity of the medium. The elastic modulus was shown to correlate positively with the turgid diameter and negatively with the circumferential stretch ratio (the ratio between turgid and plasmolysed diameter). Furthermore, cell wall thickness and elastic modulus were negatively correlated, indicating a synergistic relationship that is consistent with cell wall remodeling under external stress, corroborating results from other biophysical analyses (Hu et al., 2017).

To study the degree of variability in the parameter space, Principal Component Analysis (PCA) was performed on the input and output variables (Figures 4B and 4C). Unexpectedly, only three components account for $\sim 80 \%$ of the variability in the system, with apparent stiffness, circumferential stretch ratio, and cell wall thickness measurements providing most information gain. This indicates that the relatively simple shape of the PT does not introduce complex inter-dependencies between the input and output parameters. Furthermore, while the elastic modulus is clearly crucial to the regulation of PT growth, this analysis shows that, under our model assumptions, its variability is largely explained by other measured parameters. While the cause and effect relationship between these parameters is yet unclear, direct experimental measurements of the elastic modulus seem to be unnecessary to accurately determine PT growth rates.

\subsection{Measuring the viscosity of the cell wall}

The viscosity of the cell wall plays a central role in regulating the $\mathrm{PT}$ growth rate. Biophysical methods, such as the microindentation of static tissue to induce stress-relaxation, have previously been used to quantify vis- 
cosity (Darling et al., 2007; Hansen et al., 2011; Hayot et al., 2012). Stressrelaxation is defined as a time-dependent reduction in stress when a material is subjected to a constant mechanical strain, usually caused by rearrangements in the conformation or position of load-bearing polymer chains. This process occurs on multiple timescales corresponding to the degree and type of polymer chain displacement. The difference in viscosity between cell wall mutants is probably caused by modifying essential components of the cell wall, like pectins and $\mathrm{XyG}$, that have been shown to impact time-dependent properties (Chanliaud et al., 2002). Therefore, an accurate estimation of the relative growth rate between Arabidopsis cell wall mutants is likely to strongly depend on cell wall viscosity, which thus needs to be accurately quantified in wild-type and mutant PTs.

For the purposes of this analysis, PTs were indented with the force sensor of the CFM until a maximal force of $5 \mu \mathrm{N}$ was reached and held at this position while recording the force decay (Figure 5A). As the PTs were growing throughout this indentation procedure, the experiment faced two notable constraints compared to previous studies (Darling et al., 2007; Hansen et al., 2011). Firstly, since the growing PT is a dynamic system that absorbs wa-

Figure 4 (on the next page). Correlation and sensitivity analysis of the input and output parameters of the FEM/Monte Carlo simulations

(A) Correlogram of the parameter space including Pearson's correlation coefficients (upper triangle), histograms (diagonal), and correlation scatter plots (lower triangle) between parameters. Blue indicates a positive and red a negative correlation, respectively.

(B) Explained variance ratio of the input/output parameters based on PCA. Three components are responsible for $\sim 80 \%$ of the variance in the system.

(C) Contribution of input/output parameters to PCA components. Apparent stiffness, circumferential stretch ratio, and cell wall thickness measurements provide most information gain. 
A

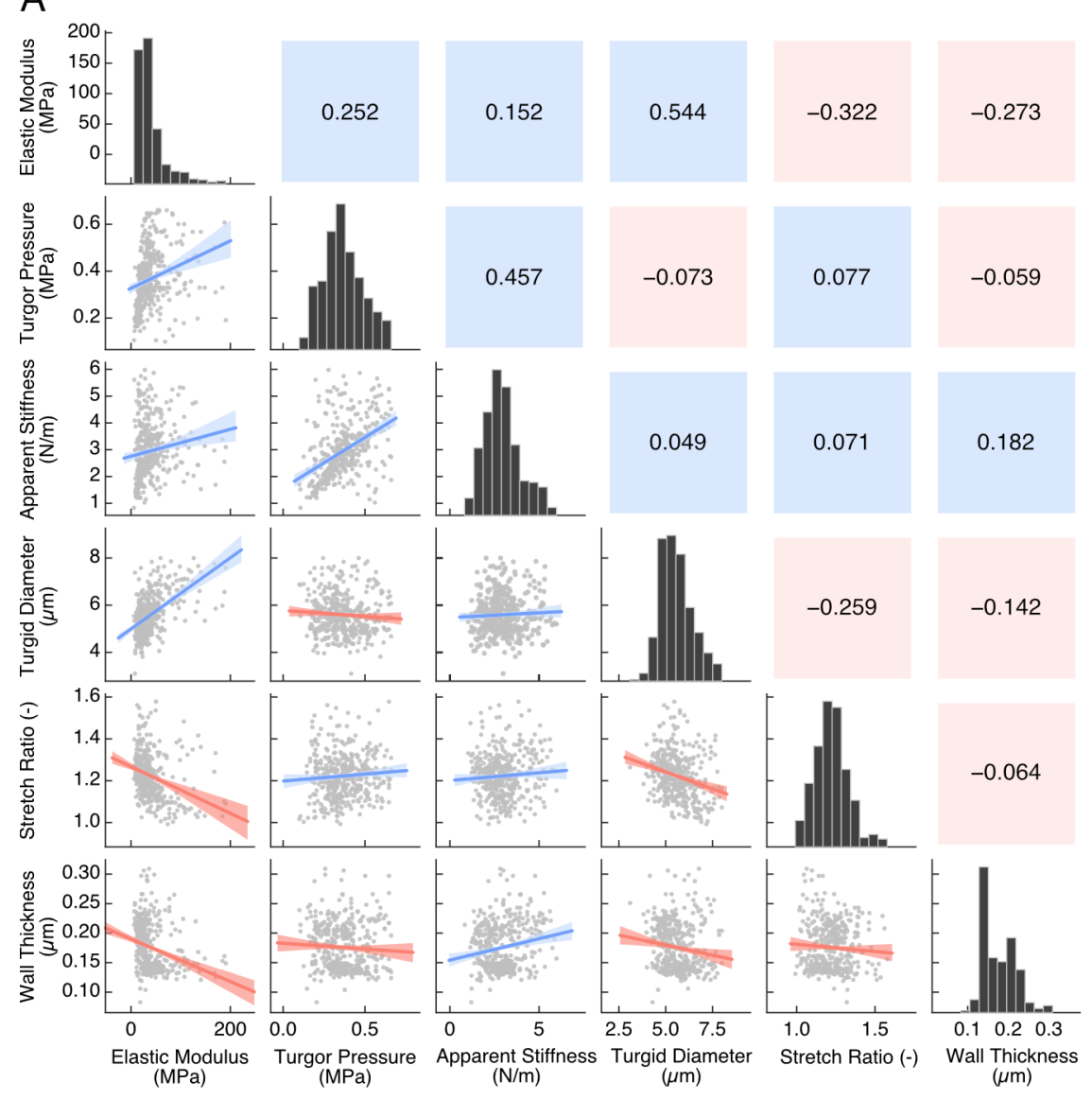

B

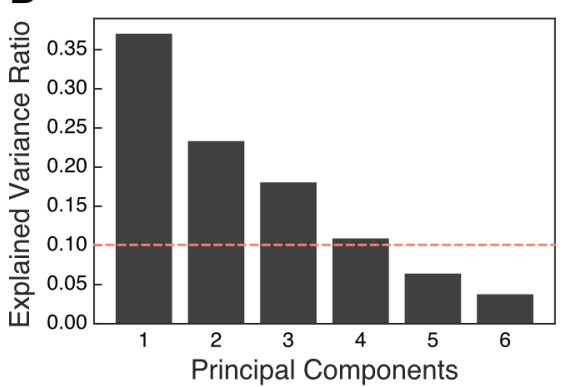

C

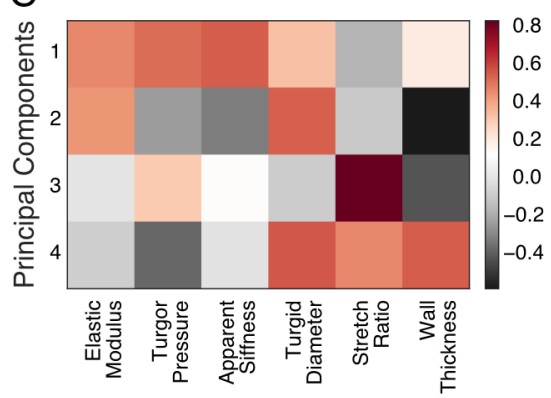


ter from the surrounding medium through osmosis (Geitmann and Ortega, 2009), we limited the applied strain duration to $5 \mathrm{~s}$ in order to avoid secondary effects that could influence the force measurements (Figure 5B). For PTs of all lines, the measured force was found to decay to a constant level in less than $5 \mathrm{~s}$, indicating that the applied strain duration was sufficient to capture the time-dependent properties relevant for PT growth (Figure 5C). To rule out measurement artifacts, a purely elastic substrate and a highly viscous glass-fiber were indented as controls using the same protocol. As expected, the force decay curves of the PTs lay within those two extremes. Secondly, as in short timescale CFM analyses, the measured force must be converted to stress to extract the time-dependent equivalent of the elastic modulus, known as the relaxation modulus. Using a simulation approach to model this experiment is not straightforward because a continuous forcing function like turgor pressure coupled with a viscoelastic cell wall renders FEM modelling unfeasible without including material plasticity to limit radial strain. However, measuring cell wall plasticity in a living and growing PT is simply impractical with currently available methods. To circumvent this constraint in estimating the relaxation modulus, we used an analytical relation developed for the nano-indentation of thin shell tubes in combination with the turgor pressure obtained from the FEM simulations. This relation is given by

$$
F=\frac{3 \pi}{4} \delta p d \phi
$$

where $F$ is the measured force, $\delta$ is the indentation depth, $p$ is the turgor pressure, $d$ is the PT diameter, and $\phi$ is a factor based on the relaxation mod- 
ulus $E^{r}$ and the cell wall thickness $h$ (Arnoldi et al., 2000; Burri et al., 2019) (see STAR Methods). This relation allows for the force $F$ to be transformed into the relaxation modulus $E^{r}$ (Figures $2 \mathrm{~F}$ and $\left.5 \mathrm{D}\right)$.

Constitutive models of biological tissues often use the generalized Maxwell linear viscoelastic model to capture relaxing systems (Macosko, 1994; Hayot et al., 2012). This model consists of a viscous damper and an elastic spring in series, the combination of which are placed in parallel with another elastic spring to describe the stress-strain behavior of a material. The relaxation modulus $E^{r}$ for such a system is given by

$$
E^{r}(t)=E^{\infty}+\sum_{i} E_{i}^{0} \exp ^{t / \tau_{i}}
$$

where $E^{\infty}$ is the equilibrium modulus at $5 \mathrm{~s}$, representing the purely elastic behavior, $E^{0}$ is the component of the elastic modulus $E$ subjected to time-dependent behavior, and $\tau=\eta / E^{0}$ is the relaxation time, where $\eta$ is the viscosity. As seen in Figure 5D, the relaxation modulus of the cell wall relaxes in two distinct phases; the initial fast decay $(t<0.5 \mathrm{~s})$ followed by the long-term slow relaxation $(t>0.5 \mathrm{~s})$, consistent with previous studies on plant tissues (Hansen et al., 2011; Hayot et al., 2012). This observation necessitates the use of two superimposed relaxation times $\tau_{i}$. However, since the slow relaxation time corresponds to the timescale of PT growth, this value, along with the equilibrium modulus, feeds the growth of the PT (Figures $2 \mathrm{G}$ and $5 \mathrm{E}$ ). From these parameters, we can estimate the viscosity of the cell wall for wild-type and mutant PTs and weigh it against the stiffness of the cell wall ( $E h, h$ being cell wall thickness) found by the elastic FEM models (Figure 5F). 
The relaxation times extracted from the stress relaxation experiments showed a large statistical dispersion between 2 and $14 \mathrm{~s}$ even though the median values for the different mutants were constrained within a relatively small interval of around 5s (Figure 5E). Some outliers are expected because, due to the inhomogeneity of the cell wall, the indenter is likely to occasionally indent local wall structures which are more pliable than the bulk cell wall material, resulting in outliers with higher relaxation times. Overall, the wild type displays the highest relaxation times, with only the pme 48 consistently showing significantly lower relaxation times. The equilibrium modulus is found to be consistent between wild-type, xxt1 xxt2, and pme $48 \mathrm{PTs}$ at $65 \%$, compared to xeg113 and xxt1 xxt2 xeg113, which returned slightly lower values. These findings indicate that the proportion of elastic-like behavior in mutant PTs are not significantly affected by their altered cell wall composition. xeg113 PTs exhibit a distinctly shaped relaxation modulus decay curve that relaxes similar to the other mutants in the initial fast decay, but returns to a wild-type-like behavior in the long-term slow relaxation phase (Figure 5D). This similarity between wild-type and xeg113 PTs is also reflected in the equivalence of their viscosities, which might indicate that, if any fluid-like structural changes are caused by the xeg113 mutation, they are adequately compensated for by other cell wall components (Figure 5F). xxt1 xxt2 and pme 48 mutants show similarly large drops in viscosity compared to the wild type, pointing to a significant increase in the fluid nature of their cell wall. The viscosity of xxt1 xxt2 xeg113 lies between xxt1 xxt2 and $x e g 113$, which may indicate a unique cell wall structure where the loss of XyGs is only partially compensated for. Indeed, a lack of XyGs seems to 
also strongly affect the elastic properties of the cell wall in xxt1 xxt2 PTs, which display a significantly lower wall stiffness than the wild type and other mutants. This seems to be the case despite $x x t 1$ xxt2 PTs possessing a thick cell wall without obvious structural defects.

\subsection{Mechanical tip growth models accurately predict growth rates of wild-type and mutant pollen tubes}

Tip growth has been studied for decades by building models predicated on assumptions with varying degrees of complexity. These models include the pre-eminent Lockhart model, based on the assumption of a viscoplastic cell wall (Lockhart, 1965), an extended version accounting for calcium dynamics, variations in cell wall thickness, and material extensibility at the tip (Kroeger et al., 2008), a further extension including the anisotropy of the cell wall

$\overline{\text { Figure } 5 \text { (on the next page). Experimental indentation results to measure cell }}$ wall viscosity

(A and B) Force-Indentation (A) and Force-Time (B) output from CFM, illustrating $5 \mathrm{~s}$ stress-relaxation on the PT by the CFM force sensor. The inset in (A) shows a closeup of the curve where the sensor is in contact with the PT. Note that the curve in the inset is corrected for the effect of the force increase while the sensor is approaching the PT. The inset in (B) shows the vertical movement of the force sensor during the experiment.

(C) Force decay (scaled by the initial force at $t=0$ ) during the retraction phase of the stress relaxation experiment on wild-type and mutant PTs and a control substrate (glassfiber).

(D) The relaxation moduli (scaled by the initial relaxation moduli at $t=0$ ) of wild-type and mutant PTs found with equation Eq. 1, exhibiting a two-phase relaxation process.

(E) Comparison of relaxation times $\tau$ and equilibrium moduli (scaled by the initial relaxation moduli at $t=0$ ) from the fitting equation Eq. 2.

(F) The viscosity derived from $\tau=\eta / E^{0}$ compared with the wall stiffness obtained from Figure $3(\mathrm{E})$. The error bars in (C) and (D) were spread apart along the time axis for better distinguishability. Originally, they would all overlap at the position of the xeg113 error bar. The whiskers in (E) and (F) represent the data between the $10^{\text {th }}$ and $90^{\text {th }}$ percentiles. The number of analyzed samples is provided at the bottom of each graph, while groups indicating significance are located at the top. 

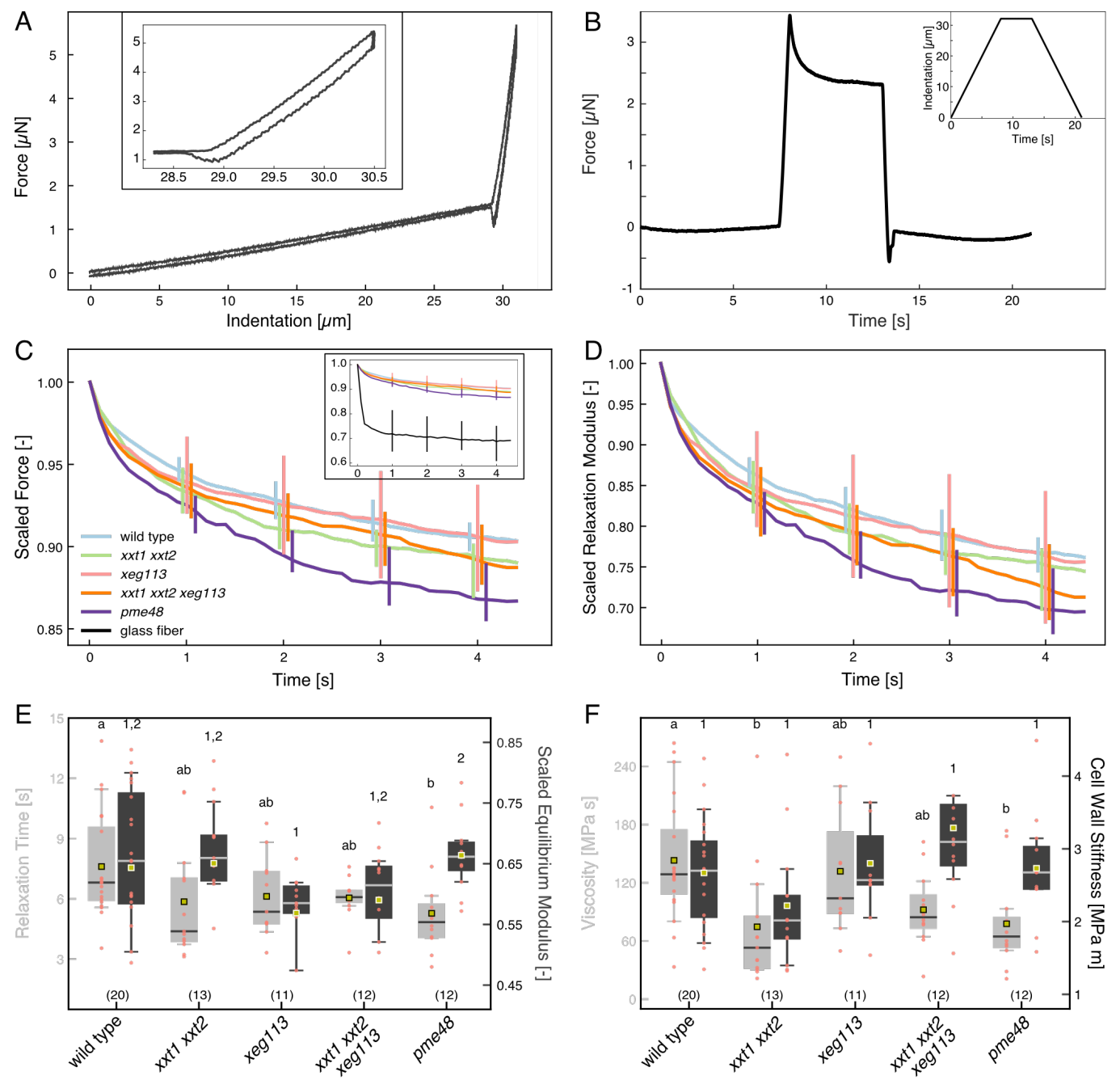
(Dumais et al., 2006), simulating spatially confined growth (Campas and Mahadevan, 2009), and a model focusing on stress-relaxation due to loss of stability (Wei and Lintilhac, 2003, 2007). The consensus among these models is that the cell wall contains both elastic and viscous components and can, therefore, be described by a linear viscoelastic constitutive law. The Lockhart model in particular utilizes the concept of a Bingham plastic fluid to describe cell wall mechanical properties. These are non-Newtonian fluids that behave like rigid bodies at low stress but flow like a viscous liquid at high stress, such as mud or mayonnaise. Applied to the cell wall, it manifests as a material that flows like a fluid (or grows uniaxially in the case of the PT) when subjected to turgor pressure above a given yield pressure, but sustains no permanent deformation at lower turgor pressures. The contribution of the elastic deformation of the cell wall to growth was later augmented to the Lockhart model (Lockhart, 1965) by Ortega and colleagues (Ortega, 1985). Assuming isotropic material properties, the strain rate of the cell wall $\dot{\epsilon}$ is described by

$$
\dot{\epsilon}=\frac{\left(\sigma-\sigma_{0}\right)}{\eta}+\frac{\partial p}{\partial t} \frac{1}{E}
$$

where $\sigma$ is the wall stress and $\sigma_{0}$ is the yield stress. Previous studies using pressure probes have indicated that the turgor pressure in Lilium longiforum PTs is stationary over time, with small pressure fluctuations proving to be uncorrelated with the growth rate (Benkert et al., 1997; Winship et al., 2010). For this analysis, we therefore assume that $\partial p / \partial t=0$. To extend the strain rate relation into the volumetric growth rate $v$ of the $\mathrm{PT}$, the $\mathrm{PT}$ tip is parametrized as a hemispherical shell with diameter $d$ as its characteristic 
length scale and wall stress $\sigma$ given by

$$
\sigma=\frac{p d}{2 h}
$$

at turgor pressure $p$ and cell wall thickness $h$. The average volumetric growth rate $v$ of the $\mathrm{PT}$ can thus be described by

$$
v=\frac{\left(p-p_{0}\right) d^{2} \kappa}{4 \eta h}
$$

where $v$ is the product of the strain rate and characteristic length, $p_{0}$ is the yield pressure, and $\kappa$ is a scaling factor. Lockhart's growth equation only captures the equilibrium growth rate, while disregarding the cytosolic calcium concentration, which has been shown to be a strong growth rate regulator, as a factor (Kroeger et al., 2011). Since we only aim to compare the relative growth rates between cell wall mutants of Arabidopsis under identical conditions, this equilibrium growth rate is sufficient for our analysis. To allow for an equal comparison of the predicted growth rate with the experimental growth rate, we introduced the scale factor $\kappa$ to correct for the effect of cytosolic calcium concentration. $\kappa$ is calculated as the ratio of the medians of predicted and experimental growth rates in the wild type and, therefore, simply normalizes the predicted and experimental growth rates for all mutant PTs. A further consideration is that PT growth cycles are known to be driven by the oscillatory nature of viscosity and cell wall thickness (Kroeger and Geitmann, 2013). However, since we are interested in the equilibrium growth rate over longer timescales, only the average viscosity and cell wall thickness as measured by the CFM and TEM experiments are 
required. Additionally, for the purpose of this analysis, the yield pressure is assumed to be constant across all cell wall mutants (Kroeger and Geitmann, 2013). Nevertheless, it should be noted that studies have shown that, unlike the other mutants, the yield pressure of pme 48 mutant PTs is more likely to diverge from the wild type because of the high concentration of pectin at the PT tip (Hepler et al., 2013).

To challenge the model prediction, we determined the growth rate experimentally by tracking $\mathrm{PT}$ growth for $10 \mathrm{~min}$ and averaging it over time (Figures 6A and 6B). The experimental growth rate of xxt1 xxt2 PTs was significantly higher than that of the wild type ( $\sim 50 \%)$, which is also reflected in the predicted growth rate (Figures $6 \mathrm{C}$ and $6 \mathrm{D}$ ). The more fluid-like nature of the $x x t 1$ xxt2 cell wall coupled with its lower cell wall stiffness provides a clear rationale for the elevated growth rate of this mutant. The similarly high growth rate ( $\sim 50 \%$ above the wild type) of xeg113 was also effectively captured by the growth model. However, since the viscosity of xeg113 is similar to that of the wild type, this growth rate elevation is more likely resulting from other factors, namely the significantly higher turgor pressure and diameters of xeg113 PTs. Despite overestimating the increase in PT growth rates compared to the wild type, the growth model predictions for xxt1 xxt2 and xeg113 mutant PTs were found to be similar to each other, mirroring their experimental growth rates. This indicates that the model is able to accurately weigh the opposing actions of viscosity, turgor pressure, and cell wall thickness on the PT growth rate. xxt1 xxt2 xeg113 PTs display the fastest experimental growth rate $(\sim 80 \%$ higher than the wild type), seemingly indicating an additive effect of the xxt1 xxt2 and xeg113 mutations. The 
predicted growth rate of xxt1 xxt2 xeg113, surprisingly, is similar to that of the wild type. While this might indicate a deficiency in the model, it should be noted that xxt1 xxt2 xeg113 PTs have a lower viscosity than the wild type, combined with the highest turgor pressure of all analyzed mutants. Therefore, the explanation for its model-predicted growth rate is based solely on its exceptionally thick cell wall. As previously stated, TEM analysis showed that $x x t 1$ xxt2 xeg113 PTs possess a thick inner cell wall with several architectural defects. Since the growth rate equation considers the cell wall to be a continuum of both the outer and inner cell walls, we re-analyzed the CFM experiments for xxt1 xxt2 xeg113 PTs considering only cell wall areas that do not show the electron-dense defects. The use of this revised cell wall thickness measurement is likely a better reflection of the growth rate of the xxt1 xxt2 xeg113 mutant, as it only includes the load-bearing constituent of the cell wall. As anticipated, the resulting predicted growth rate is much closer to the experimental growth rate (Figure 6D). The experimental growth rate of pme 48 PTs displayed a large variability as would be expected when disrupting the main constituent of the cell wall at the PT tip (Hepler et al., 2013). However, the median growth rate was $20 \%$ higher than that of the wild type, which is consistent with previous studies (Leroux et al., 2015). Furthermore, the growth equation successfully balanced the lower cell wall viscosity of pme 48 with the lower turgor pressure in the cytosol to produce an accurate prediction, with no complications arising from the yield pressure assumption. 

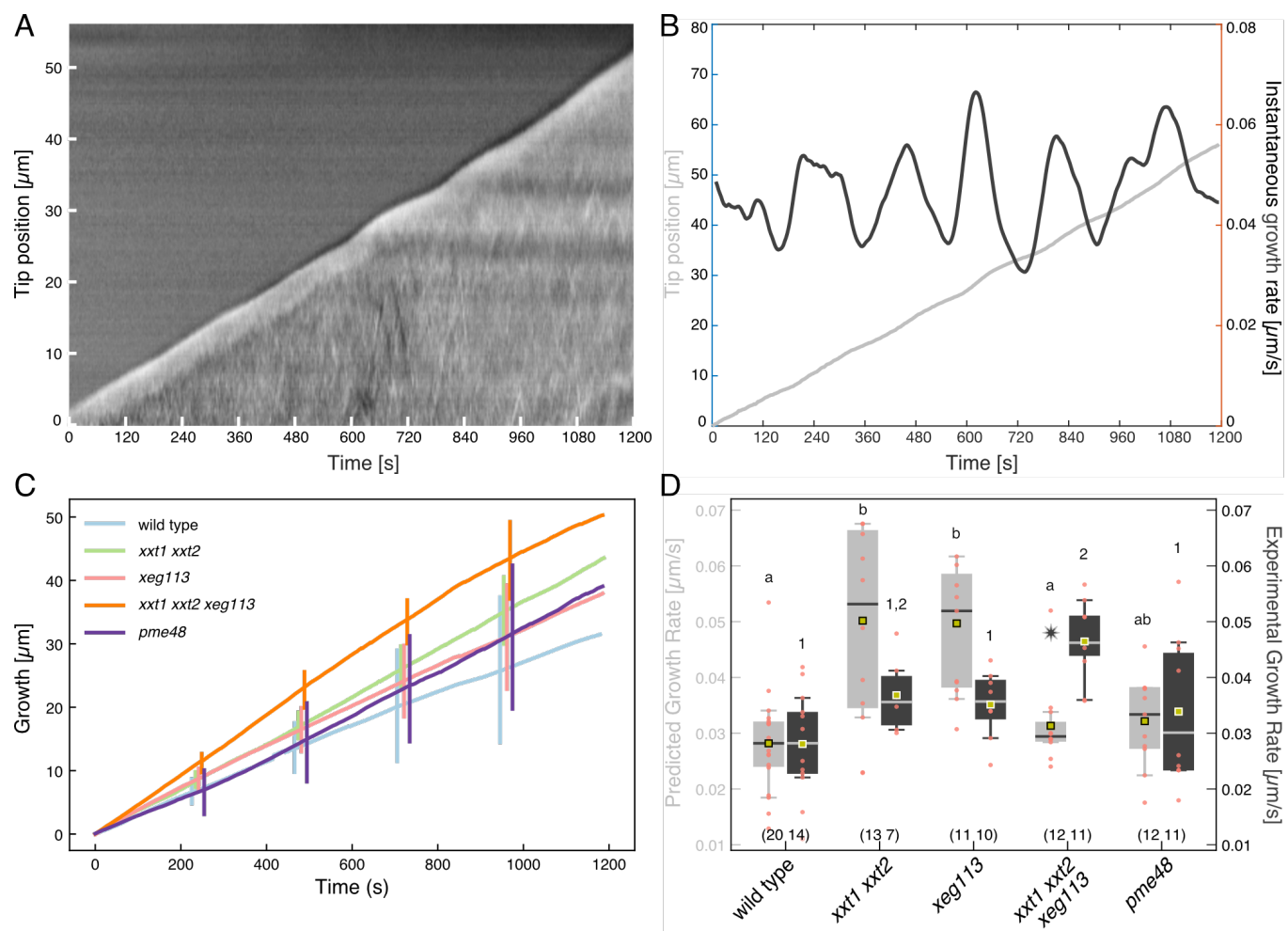

Figure 6. Growth rate analysis of wild-type and mutant pollen tubes

(A) Kymograph of an xxt1 xxt2 xeg113 PT.

(B) Tip position as a function of time and instantaneous growth rate of the PT in (A).

(C) Average measured growth of the cell wall mutants over $1200 \mathrm{~s}$.

(D) Comparison of simulated and measured growth rates of wild-type and mutant PTs. For $x x 1$ xxt2 $x e g 113$ PTs the real growth rate is substantially underestimated by the simulation. Therefore, the star marks the median predicted growth rate when a cell wall thickness of $180 \mathrm{~nm}$ for xxt1 xxt2 xeg113 PTs is assumed. See subsection 2.6 for discussion. The error bars in (C) were spread apart for better distinguishability. Originally, they would all overlap at the position of the xeg113 error bar. The whiskers of the boxplots represent the data between the $10^{\text {th }}$ and $90^{\text {th }}$ percentiles. The number of analyzed samples is provided at the bottom, while groups indicating significance are located at the top. 


\section{DISCUSSION}

The plant cell wall plays a major role in regulating cell expansion. Loosening the links between the cell wall components just the right amount allows turgor pressure to increase the cell's volume, whereas timely reinforcement of the thereby weakened cell wall matrix is necessary to avoid bursting. An imbalance between these processes either prevents cell expansion if cell wall loosening is insufficient, or leads to the bursting of the cell if the stabilisation after expansion is not adequate. Thus, a tight coordination between the external cell wall and physiological processes inside the cell is required, particularly in the vacuole, which plays a crucial role in controling turgor pressure (Kohorn et al., 2006). Therefore, a premature bursting of PTs can either be attributed to fluctuations in turgor pressure or to altered cell wall properties (Rojas et al., 2011; Kroeger et al., 2011). In order to investigate how changes in the composition of the cell wall affect its mechanical properties, we developed powerful measurement and simulation tools, which allowed us to discriminate between the similar PT growth phenotypes of several Arabidopsis mutants that have a high tendency to rupture. Time-independent indentation experiments in combination with a FEM modelling approach revealed that mutations in primary cell wall components not only affect the elasticity of the cell wall but also influence the magnitude of turgor pressure. While these two parameters were affected differently in the mutants, they generally led to a disturbance of the balance between growth-promoting and growth-limiting factors and, eventually, to the bursting of the PTs. Hence, while the wild type can effectively regulate turgor pressure and cell wall rheology to control PT growth, alterations in turgor pressure or cell wall stiffness 
in mutants with an altered biochemical composition of the cell wall drastically affect PT growth. This is especially true if mechanical barriers, such as pistilar tissues, have to be penetrated by the PT, a prerequisite for successful fertilization and, thus, seed production.

Employing FEM modelling to translate these measured parameters into usable constitutive properties was an essential step in achieving acceptable estimates for the PT growth rate; however, the Monte Carlo simulationbased uncertainty quantification was equally necessary to derive more precise outcomes. Biological measurements at the micro-scale are fundamentally subject to significant dispersion, but the inclusion of mutants that cause unpredictable growth abnormalities by disrupting mechanical equilibrium leads to markedly greater intra-mutant variability than can be reconciled by proper error estimation methods only. Despite using these techniques, the growth rate prediction of $x x t 1$ xxt2 xeg113 based solely on simulated data was considerably lower than the experimentally determined growth rate. While a closer analysis allowed us to identify a potential source of this disparity, defects that are difficult to directly quantify and capture in the models will always impact the accuracy of such analyses.

As mentioned previously, all mutant PTs had a significantly increased tendency to burst. However, this happened after a growth period of varying length, during which cell wall integrity was maintained despite irregularities, such as temporary stalling, often accompanied by swelling, branching, and leaking cytoplasm (Figure 1). Consequently, although we experimentally analysed only intact PTs, it was impossible to determine what state of stability a particular PT was in. This can explain the high variability of the 
apparent stiffness values in mutant PTs with a defective cell wall. Especially for xxt1 xxt2 xeg113 PTs, which had by far the highest bursting rate, we assume that we have overestimated the apparent stiffness as only the strongest PTs survived and, thus, could be measured.

But why would PTs that completely lack major cell wall components, such as the XyG-free $x x t 1$ xxt2 double mutant (Cavalier 2008) be able to grow at all? We assume that the cell wall integrity pathway is able to sense the deficiency in certain constituents and compensate the defects to some extent by overproducing other compounds (Voxeur and Höfte, 2016). Evidence for this hypothesis comes from immunolocalization experiments, showing that in xxt1 xxt2 PTs displaying a complete lack of XyGs, extensins were strongly overexpressed (Figure S1). Similarly, the inner cell wall of xxt1 xxt2 and xxt1 xxt2 xeg113 PTs is thickened by excessive accumulation of callose (Figures 1I, 1J, and S1). Such compensatory mechanisms are further corroborated by the observation that the lack of both XyG and functional extensins leads to synergistic phenotypic effects (Figure 1A).

Numerous studies have focused on extracting structural and hydraulic properties of PTs under specific conditions, while several others have concentrated more on their biochemical characterization. The PT growth rate provides an easily accessible framework to bridge these two perspectives, allowing to shed light onto the cause and effect relationship that exists between them. To our knowledge, this is the first study that has been able to estimate the relative growth rate among PT mutants and, thereby, to gain insights into their unique biochemical alterations and compensatory mechanisms, using solely indentation and geometric measurements. 
In summary, we integrated in vivo and in vitro measurements of $\mathrm{PT}$ growth, CFM to determine mechanical cell wall properties, and TEM analyses of the cell wall to accurately predict the growth behavior of mutants with an altered cell wall composition using FEM simulations with Monte Carlo-based uncertainty quantifications of cellular properties. This combination of experimental and modelling approaches provided novel insights into the interplay between biochemical and mechanical factors controlling cellular morphogenesis.

\section{ACKNOWLEDGEMENTS}

We are grateful to Markus Pauly (University of Dusseldorf) for providing xeg113-2 seeds, to Daniel Bollier (University of Zurich) for his ingeniosity to improve the CFM, and to Nick Jaensson for valuable suggestions on optimizing the FEM/Monte Carlo model. This work was supported by the University of Zurich, the ETH Zurich, and the Research and Technology Development Projects 'Plant Growth in a Changing Environment'(to U.G., B.J.N. and C.R.) and 'MecanX - Understanding Physics of Plant Growth' (to U.G., B.J.N., H.J.H. and C.R.), supported by SystemsX.ch, the Swiss Initiative in Systems Biology, and, in part, by an interdisciplinary grant of the Swiss National Science Foundation (CR22I2_166110 to U.G., B.J.N and H.J.H).

\section{AUTHOR CONTRIBUTIONS}

Conceptualization, U.G., C.R., B.J.N, H.J.H, H.V. and G.M.; Data curation, G.M. and H.V.; Formal analysis, G.M.; Funding acquisition, U.G., C.R., 
B.J.N. and H.J.H.; Investigation, H.V., T.N.F., C.D. and J.T.B.; Methodology, H.V., G.M., T.N.F., C.D., J.T.B. and C.E.; Project administration, B.J.N., C.R., H.J.H. and U.G.; Resources, J.P.K. and J.C.M.; Software, G.M.; Supervision, U.G., C.R., B.J.N, H.J.H, H.V., G.M. and C.E.; Visualization, H.V.; Writing - original draft, H.V. and G.M. with the help of U.G.; Writing - review \& editing, all authors

\section{DECLARATION OF INTERESTS}

The authors declare no competing interests.

\section{STAR METHODS}

\subsection{RESOURCE AVAILABILITY}

\subsubsection{Lead contact}

Further information and requests for resources and reagents should be directed to and will be fulfilled by Ueli Grossniklaus (grossnik@botinst.uzh.ch).

\subsubsection{Materials availability}

All new materials generated in this study will be available upon request from Christoph Ringli (chringli@botinst.uzh.ch).

\subsubsection{Data and code availability}

All data reported in this paper will be shared by the lead contact upon request.

All original code has been deposited at github and is publicly available as of the date of publication. 
Any additional information required to reanalyze the data reported in this paper is available from the lead contact upon request.

\subsection{EXPERIMENTAL MODEL AND SUBJECT DETAILS}

\subsubsection{Plant material and growth conditions}

All plants used were Arabidopsis thaliana (L.) Heynh of the Columbia (Col-0) accession. xxt1 xxt2 seeds (Cavalier et al., 2008) were obtained from the Nottingham Arabidopsis stock center (NASC). xeg113-2 (Gille et al., 2009) and pme48 (Leroux et al., 2015) were kindly provided by Markus Pauly and Jean-Claude Mollet, respectively. Seeds were sown on half-strength MS media (1/2 MS salt base, $10 \%$ sucrose, $0.05 \%$ MES, $0.8 \%$ Phytoagar , $\mathrm{pH}>5.7$ with $\mathrm{KOH}$ ), stratified for $2-3$ days at $4{ }^{\circ} \mathrm{C}$ in the dark, and then moved to long-day conditions $\left(8 \mathrm{~h}\right.$ dark at $18^{\circ} \mathrm{C}, 16 \mathrm{~h}$ light at $22^{\circ} \mathrm{C}$, $60 \%$ humidity). When showing two to four true leaves, seedlings were transplanted to soil and grown under long-day conditions in a walk-in growth chamber ( $8 \mathrm{~h}$ dark, $16 \mathrm{~h}$ light, $22^{\circ} \mathrm{C}, 60 \%$ humidity).

\subsubsection{Pollen tube culture conditions}

For in vitro pollen tube growth, flowers were collected and incubated for $30 \mathrm{~min}$ at $22^{\circ} \mathrm{C}$ in a moisture chamber. Liquid PTGM $(5 \mathrm{~mm} \mathrm{CaCl}, 5 \mathrm{~mm}$ $\mathrm{KCl}, 1.6 \mathrm{~mm} \mathrm{H}_{3} \mathrm{BO}_{3}, 1 \mathrm{~mm} \mathrm{MgSO}_{4}$, and $10 \%$ (w/v) sucrose, $\mathrm{pH} 7.5$ ) was prepared as described (Boavida and McCormick, 2007). Pollen grains were brushed onto silane-coated slides and covered with liquid PTGM, germinated, and grown in a moisture chamber, first at $30^{\circ} \mathrm{C}$ for $30 \mathrm{~min}$ and then at $22^{\circ} \mathrm{C}$ for at least $5 \mathrm{~h}$. For germination on solid medium, 1.5\% low melting agarose was added to liquid PTGM. For CFM measurements PTs were germinated 
on silane-coated slides.

\subsection{METHOD DETAILS}

\subsubsection{Immunocytochemical analysis of pollen tubes}

PTs grown for $5 \mathrm{~h}$ on silane-coated slides were fixed in PEM buffer $(4 \%$ paraformaldehyde in $1 \mathrm{~m} \mathrm{NaOH}, 50 \mathrm{~mm}$ PIPES, $1 \mathrm{~mm}$ EGTA, and $5 \mathrm{~mm}$ $\mathrm{MgSO}_{4}, \mathrm{pH}$ 6.9). For the enzymatic digest of selected wall components, fixed PTs were rinsed with sodium acetate buffer ( $\mathrm{pH}$ 5.5) and incubated with a $5 \mathrm{U} / \mathrm{mL}$ solution of xyloglucan-specific xyloglucanase prepared in the same sodium acetate buffer at $37^{\circ} \mathrm{C}$ for $2 \mathrm{~h}$. Enzyme-treated and non-treated fixed samples were rinsed three times with phosphate buffered saline (PBS) for 5 min each, and blocked with $5 \%$ skim milk powder (MP) in the same PBS buffer for $1 \mathrm{~h}$ or overnight at $4{ }^{\circ} \mathrm{C}$. Controls included non-digested samples and/or omitting the primary antibody. The PTs were then incubated with a 10-fold dilution of primary monoclonal Antibodies (mAbs) in PBS containing $5 \%(\mathrm{w} / \mathrm{v}) \mathrm{MP}$ for $1 \mathrm{~h}$. Samples were washed in PBS and incubated with a 100-fold dilution of fluorescein isothiocyanate-labeled secondary Ab (Sigma) in PBS/MP for $1 \mathrm{~h}$ in darkness. The samples were washed three times in PBS and mounted in a glycerol-based Citifluor AF1 anti-fade solution. Fluorescence was detected on a Leica DM6000 microscope (excitation: 480/40 nm, emission: 527/30 nm).

\subsubsection{Lyticase treatment}

For lyticase treatment, increasing concentrations (as indicated in Figure S2) of lyticase were added to the PTGM. Differential interference contrast (DIC) on a Leica DM6000 microscope was used for documentation of 
growing PTs. The length of the PTs was analysed using Fiji.

\subsubsection{TEM analysis}

A detailed description of the TEM method has been published elsewhere (Ndinyanka Fabrice et al., 2017). Briefly, PT specimens were fixed in $1.25 \%$ glutaraldehyde in $0.05 \%$ cacodylate buffer, post-fixed in $2 \%$ OsO4, dehydrated in acetone, and embedded in Epon. Then thin sections from between 5-15 m from the PT tip (corresponding to the region where CFM was performed) were collected and used for the measurement of cell wall thickness. The sections were visualized in a CM100 TEM system (FEI, The Netherlands) using a Gatan Orius 1000 CCD camera (Gatan, Munich, Germany).

\subsubsection{Determination of cell wall thickness}

The cell wall thickness of wild type and mutant PTs was determined from TEM cross sections described in subsection 7.3.3. Ten measurements around each section were averaged to give the cell wall thickness of an individual PT. All measurements were made with Fiji.

\subsubsection{Pollen tube diameter measurements}

To measure the diameter of fully turgid PTs, they were germinated and grown on silane-coated slides for 3 to $4 \mathrm{~h}$. After carefully replacing the PTGM, the PTs were left growing for another 15 to 30 min before images were taken. Afterwards, plasmolysis was induced by replacing the growth medium with $15 \%$ mannitol. Images were taken when full plasmolysis occurred, confirmed by a complete retraction of the protoplast from the tip of the PT. The diameter of turgid and plasmolysed tubes, respectively, was measured measured 
10 to $50 \mu \mathrm{m}$ behind the tip using Fiji. The mean of five measurements within this range was taken as the diameter of an individual PT.

\subsubsection{CFM measurements}

The CFM analysis was performed as described in (Vogler et al., 2013) and in (Burri et al., 2019). Arabidopsis PTs growing on silane-coated slides were visualized at a 400x magnification with DIC optics on an inverted microscope (IX 71, Olympus). MEMS-based microforce-sensors (FT-S540 and FT-S100) were used to measure the apparent stiffness and the viscoelastic relaxation of the PTs. Studies have indicated that the difference in anisotropic stresses at the tip are largely a function of the tip geometry, and even a constant isotropic elastic modulus along the pollen tube would yield different stiffness measurements at the tip compared to the shank (Vogler et al., 2013). The indentation experiments were therefore performed $10 \mu \mathrm{m}$ behind the apex and the apparent stiffness values were obtained by linearly fitting the resulting force-displacement curve. The maximal force was set to $4 \mu \mathrm{N}$, which resulted in an indentation depth of about $2 \mu \mathrm{m}$. For each PT, five measurements with four scans each were taken and >20 PT were analyzed per plant line. The resulting apparent stiffness data (Figure S3A) were used for FEM simulations. Additional parameters necessary for the analysis are listed in Figure S3 and Table 1. For the relaxation experiments, the PTs were also indented by the force probe at a distance $10 \mu \mathrm{m}$ from the tip. The position of the indenter was kept for $5 \mathrm{~s}$ after the maximal force of $5 \mu \mathrm{N}$ was reached. The force decay during the constant displacement was recoded. To determine whether the experimental setup contributes to the force relaxation, we measured a silicon cantilever (FS-C 15, SiMETRICS) that behaves in a 
purely elastic manner. In addition, we measured glass fibers, which have a geometry similar to the PTs, but a higher viscoelasticity. Data acquisition and control of the indenter were implemented in LabVIEW.

\subsubsection{Growth rate measurements}

Pollen tubes were grown in microchannels (Shamsudhin et al., 2016), and images were taken every $3 \mathrm{~s}$ on a LM using an ORCA-D2 camera (Hamamatsu Photonics K.K., Hamamatsu). KymographClear, a macro toolset for Fiji was used to produce high-quality kymographs of the growing PTs, from which the growth rate was calculated using KymographDirect (Mangeol et al., 2016).

\subsubsection{FEM simulation}

The model is inspired by an earlier first-order model that estimated the elastic modulus and turgor pressure of the PT from CFM apparent stiffness measurements using Laplace's law for thin-shell hollow tubes (Vogler et al., 2013; Young, 1805; Laplace, 1805), given by

$$
\frac{p d}{2 h}=E \log (\lambda)
$$

where $p$ is the turgor pressure, $d$ is the PT diameter, $h$ is the cell wall thickness, $E$ is the elastic modulus, and $\lambda$ is the circumferential stretch.

The driving script for the Monte Carlo simulation in Abaqus was developed in Python

The correlogram and PCA analysis plots were created in python using the sklearn and seaborn packages. 
7.3.9. Analytical relation describing the apparent stiffness for indented thin shell tubes

As described by Arnoldi and colleagues (Arnoldi et al., 2000) and established for PTs by Burri and coworkers (Burri et al., 2019), the apparent stiffness $k=F / \delta$ of a pressurized cylinder measured under indentation can be described by

$$
k=\frac{3 \pi}{4} p d \phi(\rho / \alpha)
$$

where $F$ is the measured force, $\delta$ is the indentation depth, $p$ is the turgor pressure, $d$ is the PT diameter, and $\phi(\rho / \alpha)$ is a geometric factor (Arnoldi et al., 2000; Burri et al., 2019). $\phi(\rho / \alpha)$ can be further described by

$$
\phi(\rho / \alpha)=\frac{\rho K_{1}(\rho / \alpha)}{\alpha K_{0}(\rho / \alpha)}
$$

where $\rho$ is the radius of the indenter, $K_{n}$ are modified Bessel's functions, and $\alpha$ is the cutoff distance given by

$$
\alpha^{2}=\frac{3 p d^{3}}{16\left(2 E h /\left(1-\nu^{2}\right)-p d\right)}
$$

where $E$ is the elastic modulus, $h$ is the cell wall thickness, and $\nu$ is the Poisson's ratio.

\subsection{QUANTIFICATION AND STATISTICAL ANALYSIS}

Hypothesis testing for the measured apparent stiffness and wall thickness was performed using a one-way repeated-measures ANOVA omnibus test with the application of the Greenhouse-Geisser correction due to violation of 
the sphericity property. Pairwise comparisons were then made using multiple one-way paired t-tests. Significant differences in the diameter measurements and the simulated turgor pressure and elastic modulus values were tested using a one-way ANOVA omnibus test followed by the Games-Howell posthoc test. All tests were performed with a $95 \%$ confidence interval and the pairwise comparisons used the Holm-Bonferroni correction.

\subsection{KEY RESOURCES TABLE}

Table 2. Key Resources Table

REAGENT or RESOURCE SOURCE IDENTIFIER

Antibodies

LM15

Kerafast

Cat\#ELD013

CCRC-M1

Kerafast

Cat\#EGA806

JIM5

Kerafast

Cat\#ELD004

JIM7

Kerafast

Cat\#ELD005

JIM20

Kerafast

Cat\#ELD033 
LM6

LM14

Anti-Rat IgG (whole

molecule)-FITC antibody

produced in goat

Chemicals, Peptides, and Re-

combinant Proteins

$\mathrm{CaCl}_{2}$

$\mathrm{MgSO}_{4} \cdot 7 \mathrm{H}_{2} \mathrm{O}$

Sucrose

$\mathrm{NaOH}$

Lyticase

Ethanol

Acetic acid
Kerafast

Kerafast

Sigma-Aldrich

Cat\#F6258;

RRID:AB_259695
Sigma-Aldrich Cat\#21074

Merck Millipore Cat\#105886

Panreac Ap- Cat\#A2211

plichem

Sigma-Aldrich Cat\#221465

Sigma-Aldrich Cat\#L4025

Fisher Scientific Cat\#E/0665DF/17

Merck Millipore Cat\#100063 
Chloral hydrate

$\mathrm{NaH}_{2} \mathrm{PO}_{4} \cdot \mathrm{H}_{2} \mathrm{O}$

$\mathrm{Na}_{2} \mathrm{HPO}_{4}$

Methyl blue

Citifluor ${ }^{\mathrm{TM}}$ AF1, Ein-

deckmedium

Glutaraldehyde

Cacodylic acid, sodium salt, trihydrate

$\mathrm{KCl}$

$\mathrm{OsO}_{4}$

Acetone

Epoxy-Einbettungsmittel for microscopy
Sigma-Aldrich

Cat\#15307

Panreac Ap-

Cat\#A1047

plichem

Merck Millipore

Cat\#106580

Sigma-Aldrich

Cat\#1.16316

Science Services

Cat\#E17970

$\mathrm{GmbH}$

Sigma-Aldrich

Cat\#G7651

Sigma-Aldrich

Cat\#205541

Sigma-Aldrich

Cat\#60130

Sigma-Aldrich

Cat\#75633

Scharlau

Cat\#AC03102500

Sigma-Aldrich

Cat\#45345 


\begin{tabular}{|c|c|c|}
\hline $\mathrm{H}_{3} \mathrm{BO}_{3}$ & Merck Millipore & Cat\#100165 \\
\hline $\mathrm{NaCl}$ & Roth & Cat\#3957.1 \\
\hline $\mathrm{KH}_{2} \mathrm{PO}_{4}$ & Merck Millipore & Cat\#104873 \\
\hline Rapilait skim milk powder & Migros & Cat\#150141000000 \\
\hline Phytoagar & $\begin{array}{l}\text { Duchefa Bio- } \\
\text { chemie }\end{array}$ & Cat\#P1003 \\
\hline MS salt base & Carolina & Cat\#195703 \\
\hline MES & Roth & Cat\#6066.2 \\
\hline $\mathrm{KOH}$ & Sigma-Aldrich & Cat\#P5958 \\
\hline Paraformaldehyde & Sigma-Aldrich & Cat\#F8775 \\
\hline PIPES & Sigma-Aldrich & Cat\#80635 \\
\hline EGTA & Sigma-Aldrich & Cat\#E0396 \\
\hline Na-Acetate & Sigma-Aldrich & Cat\#S2889 \\
\hline
\end{tabular}




\begin{tabular}{|c|c|c|}
\hline $\begin{array}{l}\text { Xyloglucanase (GH5) (Paeni- } \\
\text { bacillus sp.) }\end{array}$ & Megazyme & Cat\#E-XEGP \\
\hline \multicolumn{3}{|l|}{$\begin{array}{l}\text { Experimental Models: Organ- } \\
\text { isms/Strains }\end{array}$} \\
\hline $\begin{array}{l}\text { Arabidopsis thaliana: wild } \\
\text { type: accession Col-0 }\end{array}$ & $\begin{array}{l}\text { Standard acces- } \\
\text { sion }\end{array}$ & $\mathrm{N} / \mathrm{A}$ \\
\hline Arabidopsis thaliana: pme 48 : & Leroux et al., & NASC-ID: \\
\hline pme48/pme48 (Col-0) & 2015 & N622970 \\
\hline Arabidopsis thaliana: xxt1 & Cavalier et al., & NASC-ID: \\
\hline $\begin{array}{l}\text { xxt2: } x x t 1 \text { xxt2-1/xxt1 xxt2-1 } \\
\text { (Col-0) }\end{array}$ & 2008 & N16349 \\
\hline Arabidopsis thaliana: xeg113: & Gille et al., 2009 & NASC-ID: \\
\hline xeg113-2/xeg113-2 (Col-0) & & N662800 \\
\hline $\begin{array}{l}\text { Arabidopsis thaliana: } x x t 1 \\
\text { xxt2 xeg113-2: xxt1 xxt2-1 }\end{array}$ & This paper & $\mathrm{N} / \mathrm{A}$ \\
\hline xeg113/xxt1 xxt2-1 xeg113-2 & & \\
\hline$(\mathrm{Col}-0)$ & & \\
\hline
\end{tabular}

Fiji

Schindelin et al., RRID:SCR_002285 2012 
Matlab

Python Programming Lan-

guage

sklearn

seaborn

Abacus

KymographClear

KymographDirect

Chip

Other

MEMS-based microforce sen-

sors FT-S540

Silane-coated slides
RRID:SCR_001622

RRID:SCR_008394

RRID:SCR_019053

RRID:SCR_018132

RRID:SCR_013039

Mangeol et al., $\quad$ N/A

2016

Mangeol et al., $\quad$ N/A

2016

github

https://github.com/gmunglani/Chip
FemtoTools AG Cat\#FT-S540

Science Services Cat\#E63734-01

$\mathrm{GmbH}$ 


\begin{tabular}{lll} 
Lab-on-a-chip device for & Shamsudhin & N/A \\
pollen tube growth & et al., 2016 & \\
MEMS-based microforce sen- & FemtoTools AG & Cat\#FT-S100 \\
sors FT-S100 & & \\
MEMS-based microforce sen- & FemtoTools AG & no longer avail- \\
sors FT-S540 & & able \\
\hline
\end{tabular}

\section{References}

Altartouri, B., Bidhendi, A. J., Tani, T., Suzuki, J., Conrad, C., Chebli, Y., Liu, N., Karunakaran, C., Scarcelli, G. and Geitmann, A. (2019). Pectin chemistry and cellulose crystallinity govern pavement cell morphogenesis in a multi-step mechanism. Plant Physiol 181, 127-141.

Argentati, C., Morena, F., Tortorella, I., Bazzucchi, M., Porcellati, S., Emiliani, C. and Martino, S. (2019). Insight into mechanobiology: how stem cells feel mechanical forces and orchestrate biological functions. Int J Mol Sci 20, E5337.

Arnoldi, M., Fritz, M., Bäuerlein, E., Radmacher, M., Sackmann, E. and Boulbitch, A. (2000). Bacterial turgor pressure can be measured by atomic force microscopy. Phys Rev E Stat Phys Plasmas Fluids Relat Interdiscip Topics 62, 1034-1044.

Baskin, T. (2005). Anisotropic expansion of the plant cell wall. Annu. Rev. Cell Dev. Biol. 21, 203-222. 
Bedinger, P. (1992). The remarkable biology of pollen. Plant Cell 4, 879-887.

Benkert, R., Obermeyer, G. and Bentrup, F. W. (1997). The turgor pressure of growing lily pollen tubes. Protoplasma 198, 1-8.

Bidhendi, A. J. and Geitmann, A. (2016). Relating the mechanics of the primary plant cell wall to morphogenesis. J. Exp. Bot 67, 449-461.

Boavida, L. C. and McCormick, S. (2007). Temperature as a determinant factor for increased and reproducible in vitro pollen germination in Arabidopsis thaliana. Plant J 52, 570-582.

Bosch, M., Cheung, A. Y. and Hepler, P. K. (2005). Pectin methylesterase, a regulator of pollen tube growth. Plant Physiol 138, 1334-1346.

Burri, J. T., Vogler, H., Munglani, G., Läubli, N. F., Grossniklaus, U. and Nelson, B. J. (2019). A microrobotic system for simultaneous measurement of turgor pressure and cell-wall elasticity of individual growing plant cells. IEEE Robotics and Automation Letters 4, 641-646.

Campas, O. and Mahadevan, L. (2009). Shape and dynamics of tip-growing cells. Curr. Biol. 19, 2102-2107.

Cannon, M. C., Terneus, K., Hall, Q., Tan, L., Wang, Y., Wegenhart, B. L., Chen, L., Lamport, D. T., Chen, Y. and Kieliszewski, M. J. (2008). Selfassembly of the plant cell wall requires an extensin scaffold. Proc. Natl. Acad. Sci. U S A 105, 2226-2231.

Cavalier, D. M., Lerouxel, O., Neumetzler, L., Yamauchi, K., Reinecke, A., Freshour, G., Zabotina, O. A., Hahn, M. G., Burgert, I., Pauly, 
M., Raikhel, N. V. and Keegstra, K. (2008). Disrupting two Arabidopsis thaliana xylosyltransferase genes results in plants deficient in xyloglucan, a major primary cell wall component. Plant Cell 20, 1519-1537.

Chanliaud, E., Burrows, K. M., Jeronimidis, G. and Gidley, M. J. (2002). Mechanical properties of primary plant cell wall analogues. Planta 215, 989-996.

Chebli, Y. and Geitmann, A. (2007). Mechanical principles governing pollen tube growth. Functional Plant Science and Biotechnology 1, 232-245.

Cosgrove, D. J. (2016). Plant cell wall extensibility: Connecting plant cell growth with cell wall structure, mechanics, and the action of wallmodifying enzymes. J. Exp. Bot 67, 463-476.

Dardelle, F., Lehner, A., Ramdani, Y., Bardor, M., Lerouge, P., Driouich, A. and Mollet, J. C. (2010). Biochemical and immunocytological characterizations of Arabidopsis pollen tube cell wall. Plant Physiol 153, 1563-1576.

Darling, E. M., Zauscher, S., Block, J. A. and Guilak, F. (2007). A thin-layer model for viscoelastic, stress-relaxation testing of cells using atomic force microscopy: do cell properties reflect metastatic potential. Biophys. J. 92, $1784-1791$.

Dumais, J., Shaw, S. L., Steele, C. R., Long, S. R. and Ray, P. M. (2006). An anisotropic-viscoplastic model of plant cell morphogenesis by tip growth. Int. J. Dev. Biol. 50, 209-222.

Dyson, R. J., Band, L. R. and Jensen, O. E. (2012). A model of crosslink 
kinetics in the expanding plant cell wall: yield stress and enzyme action. J. Theor. Biol. 30\%, 125-136.

Eckardt, N. (2008). Role of xyloglucan in primary cell walls. Plant Cell 20, $1421-1422$.

Felekis, D., Muntwyler, S., Vogler, H., Beyeler, F., Grossniklaus, U. and Nelson, B. J. (2011). Quantifying growth mechanics of living, growing plant cells in situ using microrobotics. Micro Nano Lett 6, 311-316.

Geitmann, A. and Ortega, J. K. (2009). Mechanics and modeling of plant cell growth. Trends Plant Sci. 14, 467-478.

Geitmann, A. and Parre, E. (2004). The local cytomechanical properties of growing pollen tubes correspond to the axial distribution of structural cellular elements. Sex. Plant Reprod. 17, 9-16.

Gille, S., Hänsel, U., Ziemann, M. and Pauly, M. (2009). Identification of plant cell wall mutants by means of a forward chemical genetic approach using hydrolases. Proc. Natl. Acad. Sci. U S A 106, 14699-14704.

Hamant, O. (2013). Widespread mechanosensing controls the structure behind the architecture in plants. Curr. Opin. Plant Biol. 16, 654-660.

Hansen, S. L., Ray, P. M., Karlsson, A. O., Jørgensen, B., Borkhardt, B., Petersen, B. L. and Ulvskov, P. (2011). Mechanical properties of plant cell walls probed by relaxation spectra. Plant Physiol 155, 246-258.

Hayot, C. M., Forouzesh, E., Goel, A., Avramova, Z. and Turner, J. A. (2012). 
Viscoelastic properties of cell walls of single living plant cells determined by dynamic nanoindentation. J. Exp. Bot 63, 2525-2540.

Hayward, M. K., Muncie, J. M. and Weaver, V. M. (2021). Tissue mechanics in stem cell fate, development, and cancer. Dev. Cell 56, 1833-1847.

Hearn, E. (2001). Mechanics of materials: an introduction to the mechanics of elastic and plastic deformation of solids and structural materials. Butterworth-Heinemann, Oxford.

Hepler, P. K., Rounds, C. M. and Winship, L. J. (2013). Control of cell wall extensibility during pollen tube growth. Mol Plant 6, 998-1017.

Hu, C., Munglani, G., Vogler, H., Ndinyanka Fabrice, T., Shamsudhin, N., Wittel, F. K., Ringli, C., Grossniklaus, U., Herrmann, H. J. and Nelson, B. J. (2017). Characterization of size-dependent mechanical properties of tip-growing cells using a lab-on-chip device. Lab Chip 17, 82-90.

Iman, R. L. and Conover, W. J. (1982). A distribution-free approach to inducing rank correlation among input variables. Commun Stat Simul Comput 11, 311-334.

Iman, R. L., Helton, J. C. and Campbell, J. E. (1981). An approach to sensitivity analysis of computer models: Part I-introduction, input variable selection and preliminary variable assessment. J. Qual. Technol. 13, $174-183$.

Kim, S. J., Chandrasekar, B., Rea, A. C., Danhof, L., Zemelis-Durfee, S., Thrower, N., Shepard, Z. S., Pauly, M., Brandizzi, F. and Keegstra, K. 
(2020). The synthesis of xyloglucan, an abundant plant cell wall polysaccharide, requires CSLC function. Proc. Natl. Acad. Sci. U S A 117, 20316-20324.

Koch, A. (1966). The logarithm in biology. 1. Mechanisms generating the log-normal distribution exactly. J. Theor. Biol. 12, 276-290.

Kohorn, B. D., Kobayashi, M., Johansen, S., Riese, J., Huang, L. F., Koch, K., Fu, S., Dotson, A. and Byers, N. (2006). An Arabidopsis cell wallassociated kinase required for invertase activity and cell growth. Plant J 46, 307-316.

Krichevsky, A., Kozlovsky, S. V., Tian, G. W., Chen, M. H., Zaltsman, A. and Citovsky, V. (2007). How pollen tubes grow. Dev. Biol. 303, 405-420.

Kroeger, J. H. and Geitmann, A. (2013). Pollen tubes with more viscous cell walls oscillate at lower frequencies. Mathematical Modelling of Natural Phenomena 8, 25-34.

Kroeger, J. H., Geitmann, A. and Grant, M. (2008). Model for calcium dependent oscillatory growth in pollen tubes. J. Theor. Biol. 253, 363374 .

Kroeger, J. H., Zerzour, R. and Geitmann, A. (2011). Regulator or driving force? The role of turgor pressure in oscillatory plant cell growth. PLoS One 6, e18549.

Laplace, P. S. (1805). Traité de mécanique céleste, vol. 4,. Courcier, Paris. 
Leroux, C., Bouton, S., Kiefer-Meyer, M. C., Ndinyanka Fabrice, T., Mareck, A., Guénin, S., Fournet, F., Ringli, C., Pelloux, J., Driouich, A., Lerouge, P., Lehner, A. and Mollet, J. C. (2015). PECTIN METHYLESTERASE48 is involved in Arabidopsis pollen grain germination. Plant Physiol 16\%, $367-380$.

Limpert, E., Stahel, W. A. and Abbt, M. (2001). Log-normal distributions across the sciences: keys and clues. Bioscience 51, 341-352.

Lockhart, J. A. (1965). An analysis of irreversible plant cell elongation. J. Theor. Biol. 8, 264-275.

Macosko, C. W. (1994). Rheology: principles, measurements, and applications. Wiley-VCH, New York N.Y.

Mammoto, T., Mammoto, A. and Ingber, D. (2013). Mechanobiology and developmental control. Annu. Rev. Cell Dev. Biol. 29, 27-61.

Mangeol, P., Prevo, B. and Peterman, E. J. G. (2016). KymographClear and KymographDirect: two tools for the automated quantitative analysis of molecular and cellular dynamics using kymographs. Mol. Biol. Cell 27, $1948-1957$.

Mecchia, M. A., Santos-Fernandez, G., Duss, N. N., Somoza, S. C., BoissonDernier, A., Gagliardini, V., Martínez-Bernardini, A., Fabrice, T. N., Ringli, C., Muschietti, J. P. and Grossniklaus, U. (2017). RALF4/19 peptides interact with LRX proteins to control pollen tube growth in Arabidopsis. Science 358, 1600-1603. 
Milani, P., Gholamirad, M., Traas, J., Arnéodo, A., Boudaoud, A., Argoul, F. and Hamant, O. (2011). In vivo analysis of local wall stiffness at the shoot apical meristem in Arabidopsis using atomic force microscopy. Plant J 67, 1116-1123.

Ndinyanka Fabrice, T., Kaech, A., Barmettler, G., Eichenberger, C., Knox, J. P., Grossniklaus, U. and Ringli, C. (2017). Efficient preparation of Arabidopsis pollen tubes for ultrastructural analysis using chemical and cryo-fixation. BMC Plant Biol. 17, 176.

Ndinyanka Fabrice, T., Vogler, H., Draeger, C., Munglani, G., Gupta, S., Herger, A. G., Knox, P., Grossniklaus, U. and Ringli, C. (2018). LRX proteins play a crucial role in pollen grain and pollen tube cell wall development. Plant Physiol 176, 1981-1992.

Ngouémazong, D. E., Jolie, R. P., Cardinaels, R., Fraeye, I., Van Loey, A., Moldenaers, P. and Hendrickx, M. (2012). Stiffness of $\mathrm{Ca}^{2+}$-pectin gels: combined effects of degree and pattern of methylesterification for various $\mathrm{Ca}^{2+}$ concentrations. Carbohydr. Res. 348, 69-76.

Ortega, J. (1985). Augmented growth equation for cell wall expansion. Plant Physiol 79, 318-320.

Park, Y. B. and Cosgrove, D. J. (2012a). Changes in cell wall biomechanical properties in the xyloglucan-deficient xxt1/xxt2 mutant of Arabidopsis. Plant Physiol 158, 465-475.

Park, Y. B. and Cosgrove, D. J. (2012b). A revised architecture of primary 
cell walls based on biomechanical changes induced by substrate-specific endoglucanases. Plant Physiol 158, 1933-1943.

Park, Y. B. and Cosgrove, D. J. (2015). Xyloglucan and its interactions with other components of the growing cell wall. Plant Cell Physiol. 56, 180-194.

Parre, E. and Geitmann, A. (2005). More than a leak sealant. The mechanical properties of callose in pollen tubes. Plant Physiol 137, 274-286.

Peaucelle, A., Braybrook, S. A., Le Guillou, L., Bron, E., Kuhlemeier, C. and Höfte, H. (2011). Pectin-induced changes in cell wall mechanics underlie organ initiation in Arabidopsis. Curr. Biol. 21, 1720-1726.

Peaucelle, A., Wightman, R. and Höfte, H. (2015). The control of growth symmetry breaking in the Arabidopsis hypocotyl. Curr. Biol. 25, 17461752 .

Rojas, E. R., Hotton, S. and Dumais, J. (2011). Chemically mediated mechanical expansion of the pollen tube cell wall. Biophys. J. 101, 1844-1853.

Routier-Kierzkowska, A. L. and Smith, R. S. (2013). Measuring the mechanics of morphogenesis. Curr. Opin. Plant Biol. 16, 25-32.

Schindelin, J., Arganda-Carreras, I., Frise, E., Kaynig, V., Longair, M., Pietzsch, T., Preibisch, S., Rueden, C., Saalfeld, S., Schmid, B., Tinevez, J.-Y., White, D. J., Hartenstein, V., Eliceiri, K., Tomancak, P. and Cardona, A. (2012). Fiji: an open-source platform for biological-image analysis. Nat. Methods 9, 676-682. 
Sede, A. R., Borassi, C., Wengier, D. L., Mecchia, M. A., Estevez, J. M. and Muschietti, J. P. (2018). Arabidopsis pollen extensins LRX are required for cell wall integrity during pollen tube growth. FEBS Lett. 592, 233-243.

Shamsudhin, N., Laeubli, N., Atakan, H. B., Vogler, H., Hu, C., Haeberle, W., Sebastian, A., Grossniklaus, U. and Nelson, B. J. (2016). Massively parallelized pollen tube guidance and mechanical measurements on a labon-a-chip platform. PLoS One 11, e0168138.

Showalter, A. M. and Basu, D. (2016). Extensin and arabinogalactanprotein biosynthesis: glycosyltransferases, research challenges, and biosensors. Front Plant Sci 7, 814.

Thompson, D. (2005). How do cell walls regulate plant growth. J. Exp. Bot $56,2275-2285$.

Vogler, H., Draeger, C., Weber, A., Felekis, D., Eichenberger, C., RoutierKierzkowska, A. L., Boisson-Dernier, A., Ringli, C., Nelson, B. J., Smith, R. S. and Grossniklaus, U. (2013). The pollen tube: a soft shell with a hard core. Plant J 73, 617-627.

Vogler, H., Felekis, D., Nelson, B. J. and Grossniklaus, U. (2015). Measuring the mechanical properties of plant cell walls. Plants (Basel) 4, 167-182.

Voxeur, A. and Höfte, H. (2016). Cell wall integrity signaling in plants: "To grow or not to grow that's the question”. Glycobiology 26, 950-960.

Wei, C. and Lintilhac, P. (2003). Loss of stability - a new model for stress relaxation in plant cell walls. J. Theor. Biol. 224, 305-312. 
Wei, C. and Lintilhac, P. (2007). Loss of stability: A new look at the physics of cell wall behavior during plant cell growth. Plant Physiol 145, 763-772.

Winship, L. J., Obermeyer, G., Geitmann, A. and Hepler, P. K. (2010). Under pressure, cell walls set the pace. Trends Plant Sci. 15, 363-369.

Young, T. (1805). An essay on the cohesion of fluids. Philos Trans R Soc Lond 95, 65-87.

Zykwinska, A. W., Ralet, M. C., Garnier, C. D. and Thibault, J. F. (2005). Evidence for in vitro binding of pectin side chains to cellulose. Plant Physiol 139, 397-407. 